\title{
Object-Oriented Unsteady Vortex Lattice Method for Flapping Flight
}

\author{
Tracy E. Fritz* and Lyle N. Long ${ }^{\dagger}$ \\ The Pennsylvania State University, University Park, Pennsylvania 16802
}

\begin{abstract}
The unsteady vortex lattice method is used to model the oscillating plunging, pitching, twisting, and flapping motions of a finite-aspect-ratio wing. Its potential applications include design and analysis of small unmanned air vehicles and in the study of the high-frequency flapping flight of birds and other small flyers. The results are verified by theory and, in the plunging and pitching cases, by experimental data. The model includes free-wake relaxation, vortex stretching, and vortex dissipation effects and is implemented using object-oriented computing techniques. The results show that the method is capable of accurately simulating many of the features of complex flapping flight.
\end{abstract}

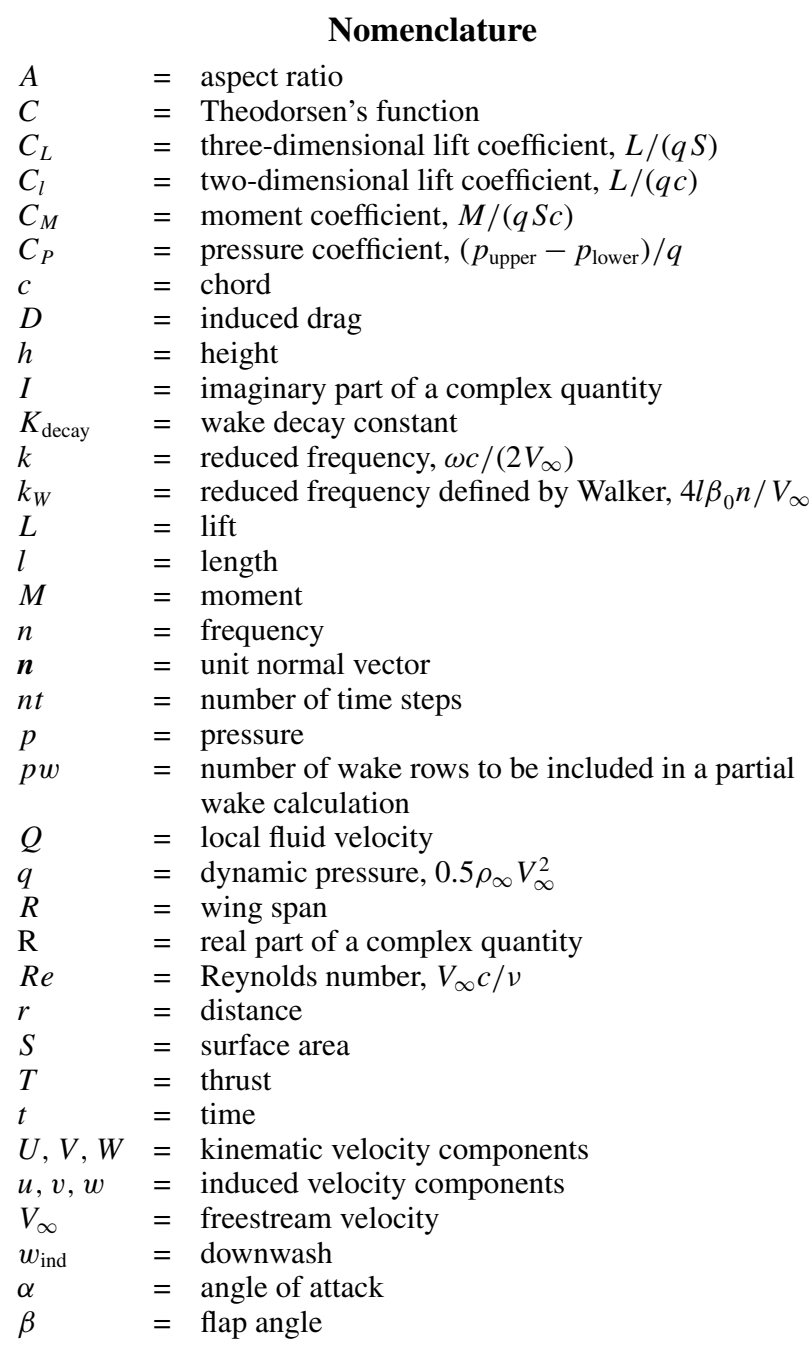

Received 31 December 2003; revision received 27 April 2004; accepted for publication 27 April 2004. Copyright (C) 2004 by Lyle N. Long. Published by the American Institute of Aeronautics and Astronautics, Inc., with permission. Copies of this paper may be made for personal or internal use, on condition that the copier pay the $\$ 10.00$ per-copy fee to the Copyright Clearance Center, Inc., 222 Rosewood Drive, Danvers, MA 01923; include the code 0021-8669/04 \$10.00 in correspondence with the CCC.

*M.S. Candidate, Department of Aerospace Engineering, 233 Hammond Building. Student Member AIAA.

${ }^{\dagger}$ Professor, Department of Aerospace Engineering, 229 Hammond Building; lnl@ psu.edu. Associate Fellow AIAA.

$\begin{array}{ll}\Gamma & =\text { circulation strength } \\ v & =\text { kinematic viscosity } \\ \rho & =\text { density } \\ \tau & =\text { tangential vector } \\ \Phi & =\text { velocity potential } \\ \phi & =\text { phase shift angle } \\ \omega & =\text { angular velocity }\end{array}$

Subscripts

ref $\quad=$ reference quantity

$W \quad=$ quantity due to the wake

\section{Introduction}

M ANY creatures in nature, as well as some manufactured devices, use flapping wings to exploit unsteady aerodynamic effects in flight. For small flying objects including bats, birds, and insects, flapping flight is an efficient technique. There has been enormous interest recently in flapping-wing micro air vehicles (MAVs), which are being built and tested with increasing success. ${ }^{1}$ Several investigators ${ }^{2-13}$ have formulated models of flapping avian flight, but progress in developing predictive tools for designing flying devices that rely on unsteady effects to achieve high-lift coefficients at low forward speeds has been limited. These models would be of interest also to those who study biological propulsion. Studying creatures in nature may help us learn how to build better MAVs, that is, biomimetics. ${ }^{14}$

Note the relevant ranges of reduced frequency $k$ and Reynolds number. Reduced frequency is a measure of the degree of unsteadiness of a problem and is usually defined in terms of the airfoil semichord $c / 2$, the angular velocity of the unsteady motion, and the freestream velocity. Reynolds number represents the ratio of inertial to viscous forces. The aerodynamics of small-amplitude wing motions with reduced frequencies below 0.1 and high Reynolds numbers $\left(>10^{5}\right)$ is well understood, as illustrated in Fig. $1 .{ }^{14}$ Steady and quasi-steady models are adequate for design and analysis of fixedwing unmanned air vehicles and large birds that fall into this category. For MAVs, Reynolds numbers may be well below $10^{5}$. Very small vehicles with low flight speeds and highly unsteady motion due to either gusts or quick maneuvers often have wings flapping with high amplitudes and frequencies. So far, work in this area has been motivated by interest in UAVs, ornithopters, ${ }^{15}$ and the flight of birds and insects. The work described herein uses an inviscid approach; thus, very low Reynolds number vehicles or insects are not considered, but the literature for a wide range of Reynolds numbers is reviewed.

The hovering flight of a hummingbird may be considered the ultimate example to model, being the only example of continuous hovering among all birds. ${ }^{16}$ Figure 2 shows a hummingbird in flight, and Fig. 3 shows the complex motions the wing undergoes. The code 


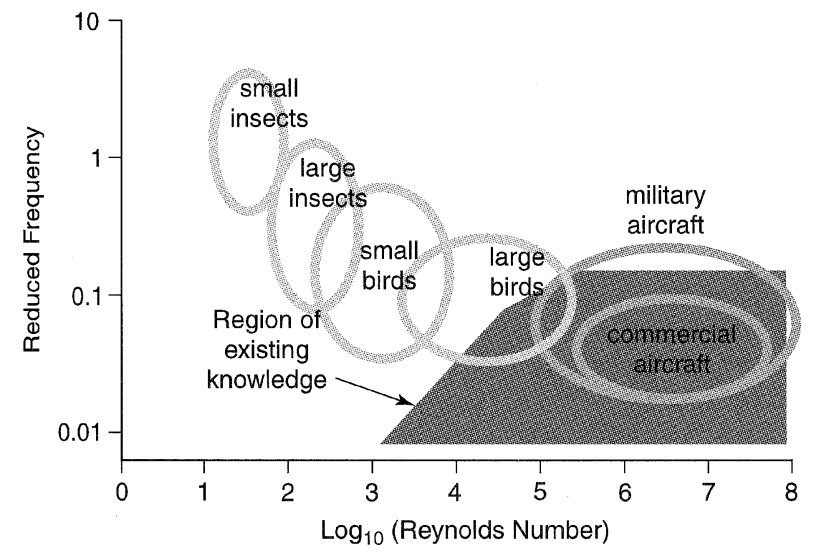

Fig. 1 Range of existing aerodynamic knowledge (Ref. 14).

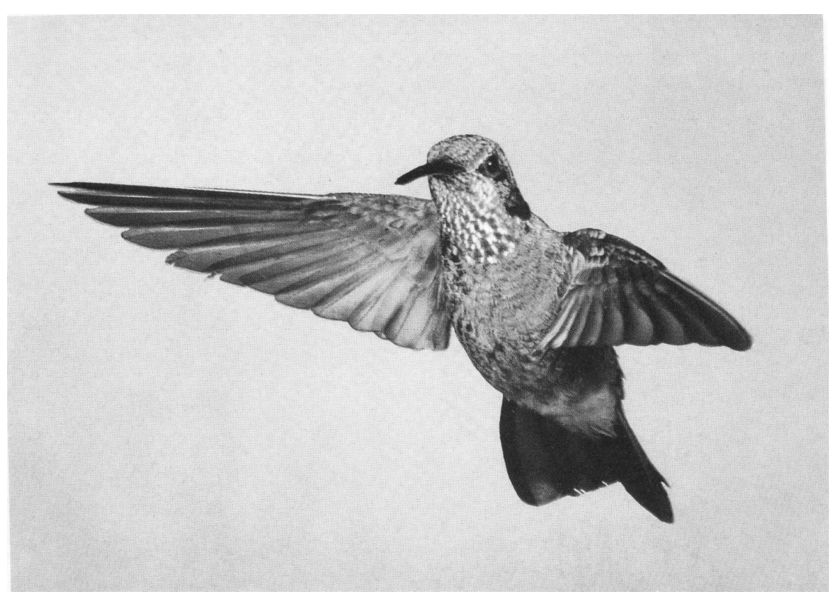

Fig. 2 Hummingbird in flight (Ref. 16).
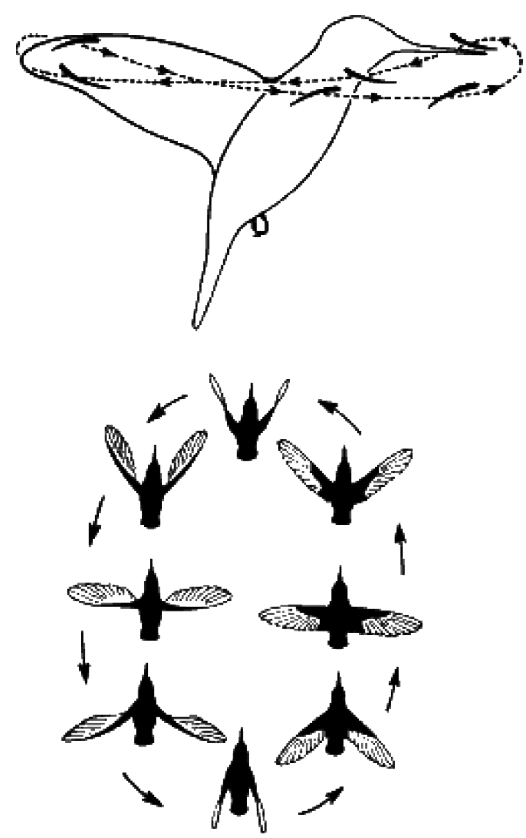

Fig. 3 Hummingbird wing motion (Ref. 6).

described herein is designed to simulate ultimately these complex motions.

An efficient object-oriented vortex lattice method (VLM) to predict the unsteady aerodynamics of flapping flight has been developed, satisfying one of the goals of this project: to create a tool to aid in understanding vehicles and creatures that produce both lift and thrust by flapping. The model is designed to capture accurately the combined effects of a free wake, flexing wings, and complex motion of the flying object A reliable numerical model for unsteady aerodynamics also has applications in rotorcraft aerodynamics and wind turbine design. ${ }^{17}$

\section{Biology-Based Work}

The flight of birds has inspired numerous theories and experiments. Much interest is generated by the seemingly impossible power and maneuverability of creatures that hover. Some biologists, for example, Pennycuick, ${ }^{18}$ have attempted to use actuator disk theory to describe the forces generated and power required by different birds, inspired by observations of brief periods of hovering. However this simple model is inadequate for flapping flight at any speed, including hovering. ${ }^{11}$ Today vortex theory is accepted as the most appropriate model to describe such unsteady aerodynamics. ${ }^{19}$ It was observed by Chai et al. ${ }^{20}$ in experiments testing the lifting capabilities of hummingbirds that changes in wingbeat amplitude were used to increase lift rather than frequency. Such observations make it clear that wing kinematics are essential to understanding the unsteady aerodynamics of flapping flight. The results also have biological implications regarding the power available in flight muscles. In another test, ${ }^{21}$ the wing kinematics were further characterized (in both biological and aerodynamic terms), the authors having noted that the wing motions of a hummingbird during hovering are highly symmetrical. They also found that, in hummingbirds, the ratio of the mass of downstroke muscle to the mass of upstroke muscle is approximately equal to two. For other birds, a typical value is 10 . This gives further insight into the mechanisms behind flapping flight at different reduced frequencies. Other experiments, ${ }^{22}$ though more biology-oriented (including studies of metabolism and energy conservation), are also important to the aerodynamicist in understanding power and efficiency concerns for highly unsteady conditions.

Others, such as Hall and Hall ${ }^{7}$ and Wang, ${ }^{5}$ have approached the problem from a different viewpoint by taking well-developed computer models of flapping flight and trying to predict optimum flapping parameters. In nature, flapping frequency selection must be based on biology and physics. However, in the design of MAVs, the application of the techniques they describe may be quite useful. Each tries to minimize (induced) power required and to maximize thrust and/or lift. Hall and Hall used a VLM with a prescribed wake. Wang's model includes shedding of a leading-edge vortex.

Flow visualization of vortex wakes can be the basis for new theories and can provide information to support or contradict existing theories. Spedding ${ }^{23,24}$ is well-known for examining the wakes of the kestrel and the jackdaw using high-speed photography. Properties of the tip vortex cores were measured (spacing and diameters) and strengths estimated and compared to lifting line theory. The estimated lift coefficient of the kestrel was approximately 1.2 at a Reynolds number of $4 \times 10^{4}$. In the second part of Ref. 23 , the wake of the kestrel was further photographed and analyzed for a flight speed of about $7 \mathrm{~m} / \mathrm{s}$ and a reduced frequency of about 0.27 . The unsteady wake was qualitatively similar to that observed in other flow visualization experiments, featuring undulating pairs of vortices. Induced power was estimated to be about $1.0 \mathrm{~W}$. Kokshaysky ${ }^{25}$ also did some wake visualization studies in an attempt to better understand the formation of vortex rings. The results were mainly qualitative. In this case, a chaffinch and a brambling flew through a cloud of dust while being photographed. Brodsky ${ }^{26}$ also visualized a vortex wake, but in the low Reynolds number regime. The wake of highly unsteady flapping creatures can be difficult to visualize whether experimentally or by simulation, which is one of the challenges in comparing simulated results to real-life phenomena.

Weis-Fogh ${ }^{2,3}$ and Weis-Fogh and Jensen ${ }^{4}$ provide a good overview and background of theories and their applicability and limitations. In Ref. 4, they critically review the theories of von Holst and Kuchemann, ${ }^{8}$ Walker, ${ }^{9}$ and Osborne, ${ }^{13}$ to determine whether the quasi-steady assumption is valid and if flapping insect flight could be explained by conventional aerodynamic principles. They explain that the von Holst and Kuchemann model is the simplest of the three, but the kinematics of the wing are oversimplified. The induced flow is neglected as well, so that the theory is reasonable for the high-speed forward flight of birds with cambered wings, that is, 
$k<0.2$, but not for low-speed or hovering flight. Weis-Fogh ${ }^{4}$ deems Walker's theory to be a bit more sophisticated in its wing kinematics, but it, too, ignores induced flow effects. Assumptions include finite forward velocity (greater than zero), constant angular velocity (equal for upstroke and downstroke), a horizontal stroke plane, and constant angle of attack during the half-stroke (though this angle of attack may be different for the upstroke and the downstroke). Osborne's theory is the most complete of the three because it successfully (according to Weis-Fogh ${ }^{4}$ ) explains insect flight without resorting to "unusual" aerodynamics. Osborne considered complex kinematics and induced flow and attempted to apply his theory to insect flight.

Betteridge and Archer ${ }^{10}$ also presented a quasi-steady model similar to those of von Holst and Kuchemann, ${ }^{8}$ Walker, ${ }^{9}$ and Osborne. ${ }^{13}$ As such, it is limited to low reduced frequencies $\left(V_{\infty} /\right.$ tip velocity $\left.>1\right)$. Man-powered aircraft, the cruising flight of birds, etc., were mentioned as applications where the theory might be useful.

Weis-Fogh ${ }^{2}$ also formulated his own expressions for average $C_{L}$, moments, power, etc., for application to flies and hummingbirds based on actuator disk/momentum theory. He investigated whether hovering was a steady or quasi-steady phenomenon over the range of Reynolds numbers. Weis-Fogh reaffirmed his previous conclusion that unsteady principles were not required to examine hovering in hummingbirds and flies, which remains debatable. Although he acknowledged that there are unsteady periods within the process of hovering, he argued that averaged results are valid. He included in these unsteady effects the idea of delayed stall, which says that there is time to build up circulation while the angle of attack is making a drastic change at the extremes of the wing stroke.

It was Weis-Fogh ${ }^{3}$ who later considered the maximum theoretical power density of biological muscle and concluded that no animal with a mass greater than approximately $100 \mathrm{~g}$ could hover continuously, though some bigger birds and bats can take off vertically and hover briefly. The discussion covered a wide range of Reynolds numbers. For a large hummingbird (Patagona gigas), the Reynolds number is around $1.5 \times 10^{4}$. For the tiny parasitic wasp Encarsia formosa, whose total body mass is $25 \mu \mathrm{g}$, the Reynolds number is 20. Weis-Fogh observed that most hoverers hover like a hummingbird, where the wings do not approach each other, angles of attack are large and positive for the forward and backward stroke, and the wing loses lift near its extreme positions, which is compensated for by the formation and shedding of vortices. His conclusions were based on observations and average $C_{L}$ calculations. He stated that "this 'normal' hovering is characterized by coefficients of lift that do not exceed the values one might expect from the steady-state aerodynamics of real wings at the relevant Reynolds numbers." ${ }^{3}$ If the observed average $C_{L}$ values of an insect seem too large, it must be because it is not hovering in this normal fashion. Vortices dissipate very quickly at low Reynolds numbers due to viscosity, so that one must turn to clap and fling, etc., ${ }^{3}$ to explain very low Reynolds number hovering.

Rayner ${ }^{11,27}$ developed a comprehensive theory for both birds and insects (including forward flight and hovering) and also dismissed momentum jet/actuator disk theory as being useless for these applications. In hover, Rayner ${ }^{11}$ describes the wake as a stack of horizontal, coaxial, circular vortex rings. In forward flight they become elliptic rings. He also noted the importance of power reduction in choosing a particular flight style. This theory is often referenced in the literature. As others before him, Rayner ${ }^{11}$ applied his ideas to real-life problems and worked out practical examples using data for real birds, but applied aerodynamic principles more rigorously than most of his predecessors. He dismissed the idea that quasi-steady theory can predict the unsteady aerodynamics of flapping flight. A rigorous treatment is given in Ref. 12.

In 1984, Ellington ${ }^{6}$ published a very complete review of existing theories and concluded that unsteady effects may very well be important for most hovering scenarios, contrary to Weis-Fogh's ${ }^{4}$ suggestion that quasi-steady aerodynamic theory is usually adequate. $\mathrm{He}^{6}$ presents a generalized vortex theory of hovering flight, including methods for estimating the mean lift, induced power, and induced velocity for unsteady and quasi-steady flight mechanisms. His series of papers includes analysis of previous theories, a discussion of morphological and kinematic parameters, a discussion of all types of aerodynamic mechanisms (steady, quasi-steady, unsteady, low Reynolds number) and a fully formulated vortex theory. This theory along with the work of Rayner, form the basis for most modern vortex theory approaches to unsteady modeling of animal flight. Together Ellington and Rayner provide a strong theoretical background for the work presented here. The reader is referred to Norberg ${ }^{19}$ for a comprehensive summary and further details.

\section{Unsteady VLM}

VLMs are well suited to the flapping flight problem (primarily at high Reynolds numbers) because they can run time accurately and can account for the changing circulation distribution on the wing, the time-dependent velocity potential, and the movement of the circulatory wake. Although they cannot model all of the physics of these problems, they may be able to predict the key phenomena. Vortex models are widely used in aircraft and rotorcraft analysis, partly because computational fluid dynamics (CFD) models ${ }^{28}$ are much more computationally expensive. CFD is simply not currently feasible for flapping flight.

The basics of the unsteady VLM are described in Ref. 29. Vortex rings are arranged in a grid on a lifting surface. As the surface moves through the fluid, the circulation of these rings changes and a row of rings is shed from the surface's trailing edge at each time step. Once the rings are shed, they become part of the wake, and their circulation does not change (unless dissipation is included in the model). Some models prescribe, in advance, the motion of the wake based on empirical data, or simply leave the wake rings where they were at the time of shedding, ${ }^{7}$ though strictly speaking, each ring in the wake should move with the local flow velocity. This local velocity is a combination of the velocity induced by the other rings in the wake and the rings bound to the surface and the freestream velocity. The strength of the circulation of the rings bound to the surface is determined by imposing the zero normal flow boundary condition on the surface, where the flow at the surface is a combination, again, of the velocity induced by the wake rings, the velocity induced by the rings bound to the surface, and the freestream velocity.

The Biot-Savart law for a finite vortex segment is

$$
\mathrm{d} \boldsymbol{v}=(\Gamma / 4 \pi)\left[(\mathrm{d} \boldsymbol{l} \times \boldsymbol{r}) / r^{3}\right]
$$

where $\mathrm{d} v$ is the incremental velocity induced by a vortex segment of incremental length $\mathrm{d} l$. The strength of the circulation is denoted by $\Gamma$ and $\boldsymbol{r}$ is the distance from the segment to the point where the velocity is to be determined. By adding the effects of each segment in a ring the velocities induced by each ring can be determined. These can be combined to compute the effect of every ring on every other ring. Then the equation

$$
\left(\begin{array}{cccc}
a_{11} & a_{12} & \cdots & a_{1 m} \\
a_{21} & a_{22} & \cdots & a_{2 m} \\
a_{31} & a_{32} & \cdots & a_{3 m} \\
\vdots & \vdots & \vdots & \vdots \\
a_{m 1} & a_{11} & \cdots & a_{m m}
\end{array}\right)\left(\begin{array}{c}
\Gamma_{1} \\
\Gamma_{2} \\
\Gamma_{3} \\
\vdots \\
\Gamma_{m}
\end{array}\right)=\left(\begin{array}{c}
R H S_{1} \\
R H S_{2} \\
R H S_{3} \\
\vdots \\
R H S_{m}
\end{array}\right)
$$

must be solved at every time step for the circulation strength of the rings bound to the surface. The influence coefficients $a_{K L}$ are evaluated using a unit strength vortex,

$$
a_{K L}=(u, v, w)_{K L} \cdot \boldsymbol{n}_{K}
$$

where $a_{K L}$ is the influence of the $L$ th panel's unit strength vortex ring on the $K$ th panel, that is, the velocity normal to the $K$ th panel induced by the $L$ th panel. (Here $\boldsymbol{n}_{K}$ is the unit normal vector of the $K$ th panel.) The right-hand-side (RHS) $K$ values, $\mathrm{RHS}_{K}$ are calculated for each ring by

$$
\operatorname{RHS}_{K}=-\left[U(t)+u_{W}, V(t)+v_{W}, W(t)+w_{W}\right]_{K} \cdot \boldsymbol{n}_{K}
$$


where $U, V$, and $W$ are is the time-dependent kinematic velocities of the wing and $(u, v, w)_{W}$ is the local velocity induced at the $K$ th panel by the entire wake.

Because the system of linear equations must be solved at every time step, an efficient linear algebra algorithm is essential. Also, if the surface geometry changes with time, then the matrix of influence coefficients changes and must be reformed (and resolved) at every time step. When the matrix changes often, methods such as lower-upper (LU) decomposition might not be optimal, and iterative schemes might be more effective. Complex wing motions can also require additional work to apply rotation matrices.

For a complex surface, a large number of rings may be needed to form an accurate matrix of influence coefficients. It is well known that direct methods, such as LU decomposition or Gaussian elimination require $\mathcal{O}\left(N^{3}\right)$ operations, where $N$ is the number of rings on the wing. Iterative methods, such as conjugate gradient (CG) or biconjugate gradient stabilized ${ }^{30}$ may be more suitable, depending on the form of the matrix. In the method described here, either LU decomposition or CG is used, and the CG method can be run in either serial or parallel mode using the message passing interface library. ${ }^{31}$

Once the new values for circulation on the wing have been found, the aerodynamic loads can be computed. To find the pressure distribution and, hence, the wing's lift coefficient, the unsteady Bernoulli equation is used:

$$
\frac{p_{\mathrm{ref}}-p}{\rho}=\frac{Q^{2}}{2}-\frac{v_{\mathrm{ref}}^{2}}{2}+\frac{\partial \Phi}{\partial t}
$$

where $Q$ is the local fluid velocity (including kinematic and induced velocities), and the subscript ref indicates far-field reference conditions. In terms of local circulations, the pressure jump across an individual panel is then given by ${ }^{29,32}$

$$
\begin{aligned}
\Delta p_{i j} & =\rho\left\{\left[U(t)+u_{W}, V(t)+v_{W}, W(t)+w_{W}\right]_{i j}\right. \\
& \times \tau_{i} \frac{\Gamma_{i, j}-\Gamma_{i-1, j}}{\Delta c_{i j}}+\left[U(t)+u_{W}, V(t)+v_{W}, W(t)+w_{W}\right]_{i j} \\
& \left.\times \tau_{j} \frac{\Gamma_{i, j}-\Gamma_{i, j-1}}{\Delta b_{i j}}+\frac{\partial}{\partial t} \Gamma_{i j}\right\}
\end{aligned}
$$

where $\boldsymbol{\tau}_{i}$ and $\boldsymbol{\tau}_{j}$ are the panel tangential vectors in the $i$ and $j$ directions and $\Delta c$ and $\Delta b$ are the panel chord and span, respectively. The first two terms represent the chordwise and spanwise components of the tangential velocity due to the wing vortices, respectively. The third term represents the velocity potential time derivative. This third term is very important as discussed later, for accurately simulating flapping flight at high reduced frequencies.

A panel's contribution to induced drag is given by

$$
\Delta D_{i j}=\rho\left[\left(w_{\text {ind }}+w_{W}\right)_{i j}\left(\Gamma_{i j}-\Gamma_{i-1, j}\right) \Delta b_{i j}+\frac{\partial}{\partial t} \Gamma_{i j} \Delta S_{i j} \sin \alpha_{i j}\right]
$$

where $w_{\text {ind }}$ is the induced downwash at each ring, $\alpha$ is the panel's angle of attack, and $\Delta S$ is the surface area of the panel. The value of $w_{\text {ind }}$ is the sum of the velocities induced by all of the chordwise segments of the vortex rings bound to the wing, the calculation of which is already performed in finding the matrix of influence coefficients.

The wake model used here accounts for free relaxation as well as wake aging (dissipation) and vortex stretching. The various wake features can be selected independently by the user so that various combinations of wake effects can be tested. The free relaxation of the wake can be approximated by a prescribed or frozen wake in the case of high-speed forward flight where the wake is quickly left behind the wing and its influence on the wing quickly becomes negligible. However, because our ultimate goal is to model highly unsteady hovering vehicles and/or birds, it is important to preserve the physical accuracy of a free wake. In such cases, the wake will

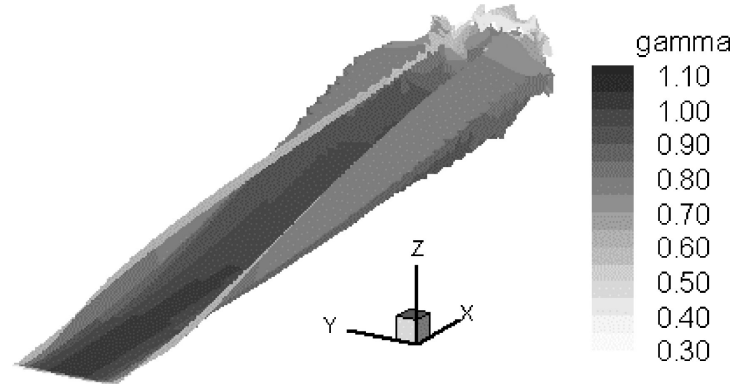

a)
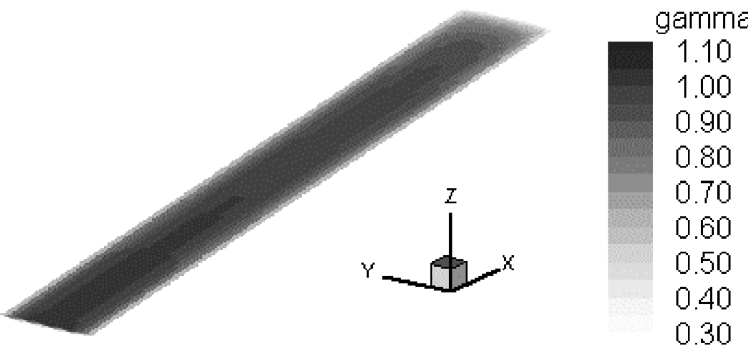

b)

Fig. 4 Plunging wing wake: a) free and b) fixed.

remain near the wing and the shed wake rings will continue to have an influence on the vehicle's aerodynamics for a long time. Calculating the velocity at every point on the wake, which includes calculating the influence of every other wake ring plus the influence of the wing rings, is computationally expensive and time consuming, and so the user can choose to include the influence of any number of rows of the wake. Of course, more sophisticated approaches, such as a multipole method, ${ }^{33-35}$ could also be implemented.

The free wake cannot support any load and, thus, moves from its original position with the local velocity. The velocity field behind the wing or vehicle is not constant, and so the vortex rings in the wake become stretched and distorted. The code accounts for these effects by redistributing the circulation along the changing perimeter of each ring at every time step. In this way the total circulation of each ring in the wake is preserved while the accuracy of the influence of each segment of the four-sided ring is not over- or underestimated as its length changes. The object-oriented nature of the code makes this a simple task because each ring segment keeps track of its own current length, original length, etc. Figure 4a shows the wake (and circulation distribution on the wake) of a cambered airfoil plunging steadily at an angle of attack of $6 \mathrm{deg}$. In this case, free relaxation with wake stretching is enabled. Note that the wake far behind the wing becomes somewhat chaotic and has decreasing influence relative to the newer wake. The rolling up of the wake into two tip vortices is also visible. In Fig. 4b, the same wing undergoing the same motion is shown with a fixed wake, that is, the wake stays where it was when it was initially shed.

The program is also capable of modeling wake aging or wake decay with time. The circulation of the wake rings may in reality decrease with time as a result of viscous dissipation or turbulence, and any one of various models could easily be implemented within the code. The vortex ring model consists of filaments represented as finite-core Rankine vortices, where the core is treated as a solidbody rotation, and outside the core, the induced velocity decreases hyperbolically according to potential theory. The approach to aging involves reducing the peak velocity of the vortex as the square root of time. ${ }^{36}$ The circulation of any ring in the wake is modeled by

$$
\left.\Gamma=\Gamma_{0} \sqrt{K_{\text {decay }} /\left[\left(V_{\infty} t / c\right)+K_{\text {decay }}\right.}\right]
$$

where $\Gamma_{0}$ is the initial value of circulation, $t$ is time since the ring was shed into the wake, $c$ is wing chord, and $K_{\text {decay }}$ is a constant parameter that reflects the rate at which the circulation in the wake should decay. Figure 5 shows a case with no wake aging compared to the same case with $K_{\text {decay }}=60$. 


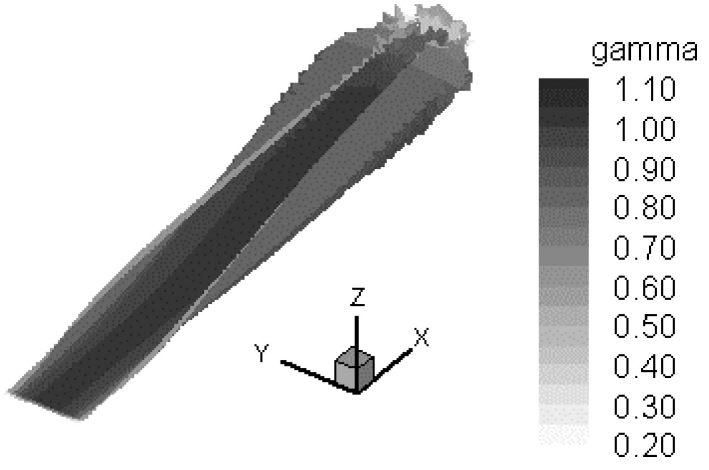

Fig. 5a Wake aging disabled; $\alpha=6 \mathrm{deg}$, cambered wing, $A=8$.

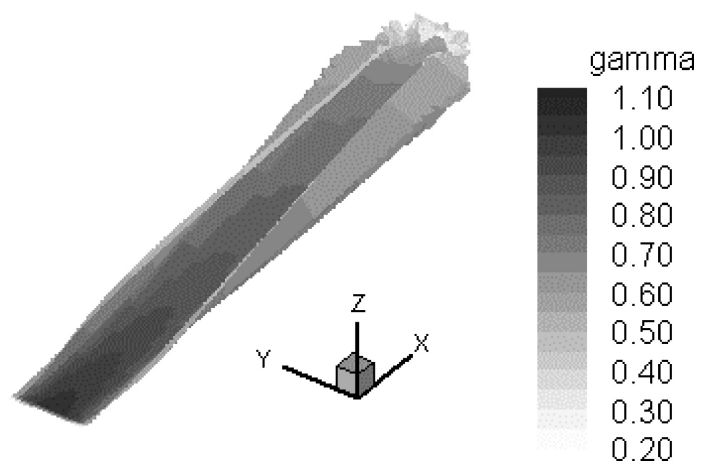

Fig. 5b Effect of wake aging parameter $K_{\text {decay }}=60$ on the plunging wing from Fig. 5 a.

A value of 60 was chosen so that for the number of time steps run, the circulation of the oldest rings should have been reduced from their initial value (when first shed into the wake) by approximately $30 \%$. Leishman ${ }^{36}$ shows that, for a helicopter rotor, the peak velocity should be reduced by about $65 \%$ after 250 chords of travel. This quantitative reduction is not easily verifiable by examining Fig. 5, but the qualitative picture is clear. The circulation contour has obviously changed, but also note that the degree to which the tip vortices roll up is lessened.

For cases where the oldest parts of the wake are far away from the wing and have little influence on the wing's aerodynamics, they can be ignored in the load calculations. The option to ignore a chosen number of old wake rows (beginning with the oldest row) is useful because the calculation of the local velocities everywhere on the wake is a compute-intensive task. These rows are not completely ignored because their influence is still included in the calculation of local velocity elsewhere on the wake. However, they are not moved with local velocity, and their influence on the wing is ignored. Another similar option is available where these old rows of wake are removed completely, saving computer memory and run time. In this case, their influence on the rest of the wake, as well as on the wing, is completely ignored.

As a simple example to illustrate the potential time savings, the code was run on a $1.8-\mathrm{GHz}$ Pentium 4 computer for 10 time steps. The number of rows of the wake to be included in the calculation was five. The two most important steps in the code affected by this option are the calculation of the local velocities everywhere on the wake, and the formation of the $\{$ RHS $\}$ vector from Eq. (1), which includes contributions from the entire wake. Figure 6 shows how the time required for each of these steps is affected by reducing the number of wake elements used.

The change in the aerodynamics due to ignoring old parts of the wake is minimal, whereas the savings in CPU time is considerable. The wake for a case with sinusoidal plunging oscillation at a reduced frequency $k$ of 0.25 is shown in Fig. 7 .

For the case in Fig. 7a, the full wake was included in all calculations for each of the 780 time steps. For the case in Fig. 7b, the oldest 160 wake rows were only used to compute induced velocities on the other 620 rows of wake (the wing moving approximately three span

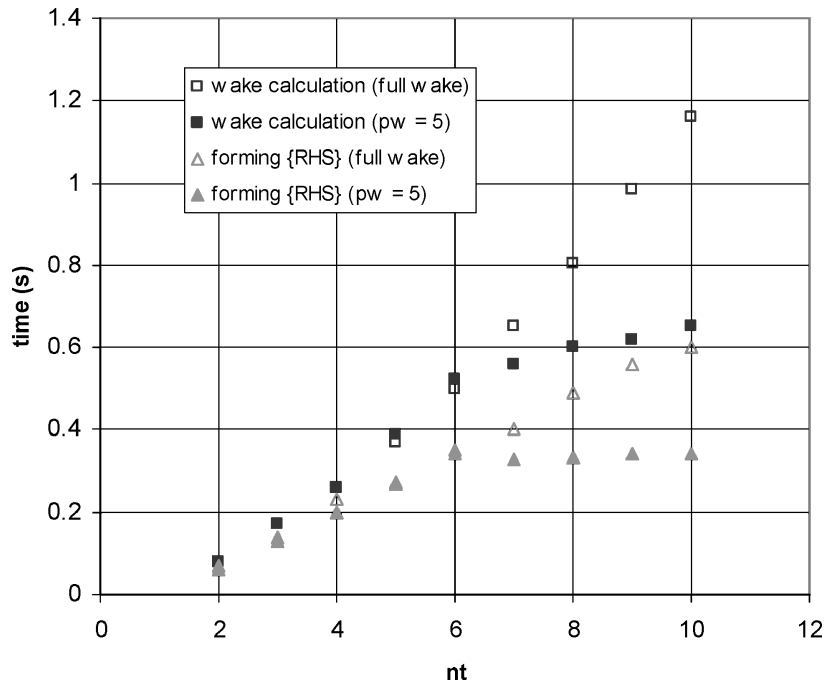

Fig. 6 Effect on CPU time of excluding part of the wake in calculations.

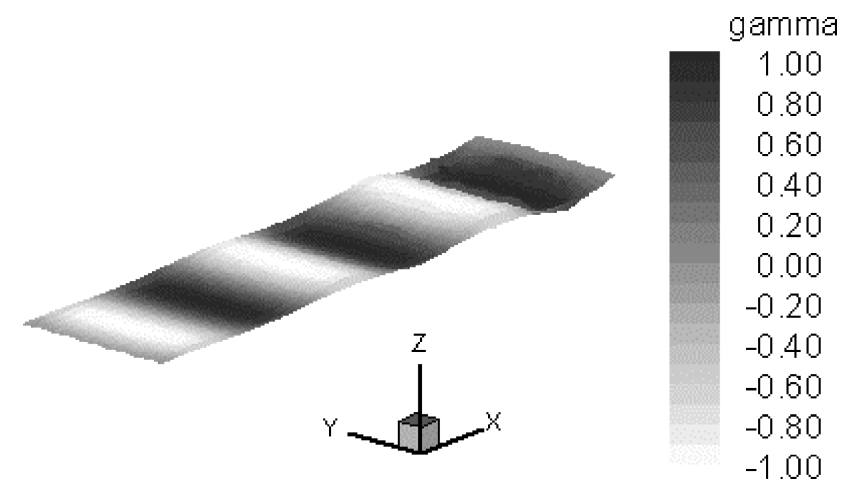

a)

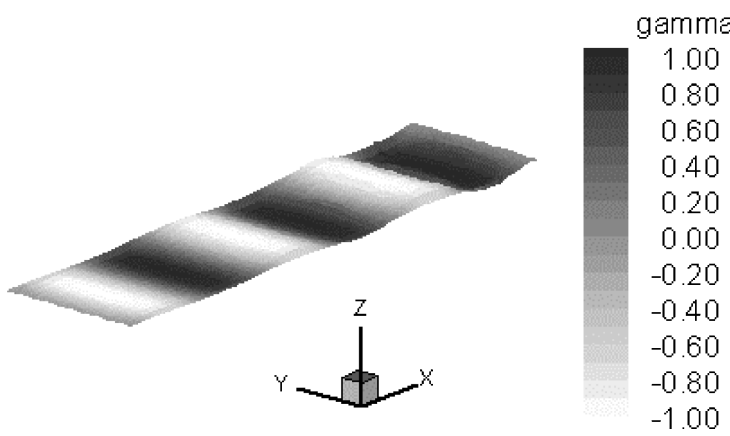

b)

Fig. 7 Pure plunging oscillation, $k=0.25$ : a) full wake included in calculations and $b$ ) effect on wake of $p w$ parameter.

lengths after 620 time steps), and they themselves were not moved. Therefore, differences in wake shape and calculated aerodynamic loads should appear only in the oldest $21 \%$ of the wake and latest $21 \%$ of the time-dependent lift curve, respectively. Figure 7 shows that the two wakes are nearly identical. As expected, the $C_{L}$ vs time curves are identical up to $79 \%$ of the total time. Afterward, the difference in the lift coefficients did not exceed $0.5 \%$. As the wake grows, the CPU time increases due to the larger number of vortex rings. During the time when the entire wake is included in the calculations, that is, time steps $<620$, the rate of change of time required to calculate the local velocities over the wake was $1.25 \mathrm{~s}$ per time step. Beyond 620 time steps, the rate of increase was reduced to $0.64 \mathrm{~s}$ per time step. This means that the wake velocity calculation time alone decreased by $49 \%$ over the last 160 time steps. The number of wake rows that should be included may depend on the reduced frequency. These improvements are understandable because many of the computations involved here are either $\mathcal{O}\left(N^{2}\right)$ or $\mathcal{O}\left(N^{3}\right)$. 


\section{Object-Oriented Implementation}

This code follows a highly object-oriented approach and is written in $\mathrm{C}++$. Such implementations make the code more readable, maintainable, portable, and extensible. ${ }^{37}$ The program is also efficient due to $\mathrm{C}++$ memory management features and the use of pointers. The concepts of $\mathrm{C}++$ classes and class inheritance help make the code easy to use and modify.

A large vortex lattice is made up of many individual rectilinear rings, and each ring is broken into vortex filaments. This approach lends itself very naturally to an object-oriented approach. The expression shown earlier for velocity induced at a point by a vortex filament of finite length is relatively simple, as given by the BiotSavart law, compared to what it might be for a ring. Through the use of a vortex filament class of objects, each filament in the lattice can be made responsible for calculating and keeping track of its own properties. This information is known only to each filament, that is, it is a private member of the filament class. This is referred to as encapsulation. Such a class can send its information to other types of objects that may require its data, although outside objects may not alter directly another object's private data members.

The ring objects contain filament objects, plus additional data that are not required by filaments, such as a normal vector. A ring need only keep track of which elements belong to it and can then indirectly pass along the location of its corner points. The next level of organization is the lattice of rings, which may represent either a surface or a wake. Though both represent a rectilinear grid of rings, a collection of surface rings has different properties than a collection of wake rings. Here, $\mathrm{C}++$ inheritance makes it easy for two derived classes, wake and wing, to share common properties of the parent class, lattice, while retaining their own distinct features (distinct data and methods). This eliminates the need for a large number of variables with cryptic names and also allows for sensible, descriptive function naming so that the code is easier to read and understand. It also avoids the proliferation of multidimensional arrays as in FORTRAN.

The $\mathrm{C}++$ class concept can also be applied in more abstract ways. For example, there is an object that is used to solve linear systems of equations and an object for writing output data files. In this way, specific aspects of the code can be modified without risk of disrupting other parts of the code or changing the structure of other types of objects. This also encourages code reuse because users can use the code without worrying about the details of the inner workings. In addition, the code can be modified and improved without affecting the user because the user does not rely on the inner details.

When these objects are used, the unsteady VLM code itself flows in a logical sequence that is easy to understand. The responsibility of the detailed calculations is contained in the individual objects. First, the geometry of the surface is defined and discretized into a rectangular grid of panels. For each panel, rings are created, and these are formed into a lattice. Then the matrix of influence coefficients is determined based on the surface geometry. These influence coefficients are found at the beginning of each time step. The strengths of the vortex rings on the surface are solved for by applying the zero normal flow boundary condition as described earlier.

Once the aerodynamic loads and other important parameters have been calculated for the surface (the wing), the surface must be moved and deformed according to a prescribed motion for the next time step. A new influence coefficient matrix is formed, and the process repeats. An advantage of making the code object oriented is that other surfaces can be added to the simulation with ease because the functions and variables necessary to create and manipulate them are already in place. One could even model the interactional aerodynamics of two birds flying near one another, or main rotor and tail rotor interactions during complex helicopter motions.

\section{Results}

In this section several sets of results from the earlier described computer program are presented. The code was systematically used to simulate increasingly complicated cases. Initially, an impulsively started wing in rectilinear motion was simulated. The code was then

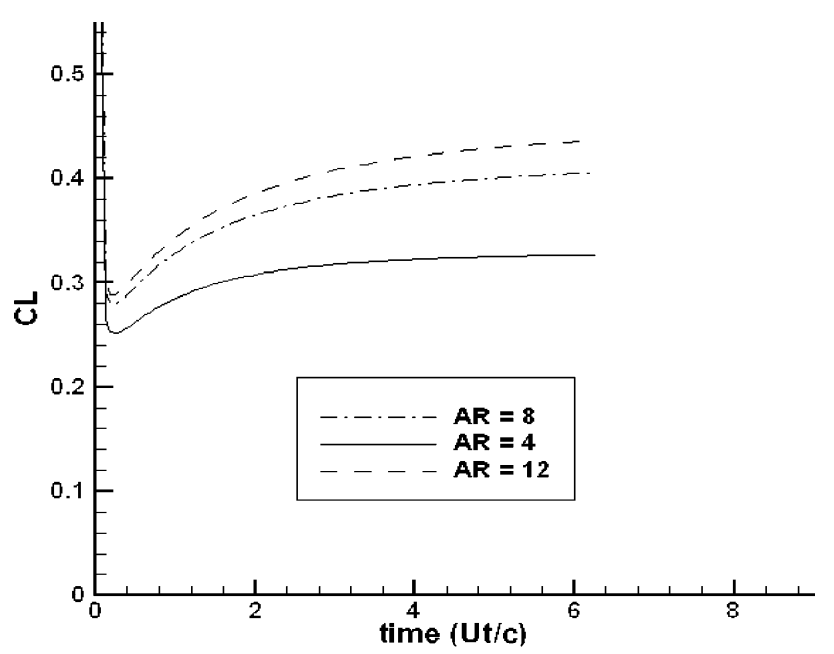

Fig. 8 Transient lift coefficient for a rectangular plunging wing.

applied to a simple wing in both plunging and pitching harmonic motion and compared to theory and experiment. The next set of results presented are for a wing in forward flight with a simple flapping motion. Finally, results will be presented that show wings in forward flapping flight that also have spanwise varying dynamic twist. All of these results are quite promising.

\section{Steady-State Flight}

For the simple case of a rectangular wing plunging in quiescent air, the transient lift coefficient matches the predictions found in Ref. 29 for several aspect ratios. Figure 8 shows three cases where the nondimensional time step $\left(V_{\infty} \Delta t\right) / c$ is equal to $1 / 16$. The complicated shape of the curves represents the superposition of an unsteady and a steady part. The unsteady part quickly decays, leaving the steady-state solution as time becomes large.

\section{Pitching and Plunging Oscillations}

To further validate the code, simulations of harmonically pitching and plunging wings were also performed. A large amount of data for various unsteady cases is presented by Halfman. ${ }^{38}$ Lift and moment data for simple harmonic oscillatory plunging and pitching cases and combinations of the two are provided. Experimental data are also compared to Theodorsen's theory. ${ }^{39}$

Halfman's experimental apparatus ${ }^{38}$ consisted of a symmetric (NACA 0012) airfoil (with a chord of $1 \mathrm{ft}$ or $0.3048 \mathrm{~m}$ ) spanning the wind-tunnel walls to simulate an infinite aspect ratio. For the simulations, an aspect ratio of 10 was sufficiently large to duplicate the data. For the plunging cases, the wing (at zero angle of attack) oscillated according to $h=h_{0} \sin (\omega t)$ in a constant freestream velocity. Values of maximum amplitude $h_{0}$ of 1 and 2 in. (2.54 and $5.08 \mathrm{~cm}$ ) were tested. Values of $\omega$ were deduced from the given reduced frequency for each data point, where $\omega=\left(2 k V_{\infty}\right) /$ chord. Values of $k$ ranged approximately from 0.05 to 0.4 . For the pitching cases, $\alpha$ oscillated according to $\alpha=\alpha_{0} \sin (\omega t)$, also at a constant $V_{\infty}$. Values for $\alpha_{0}$ of 6.74 and $13.48 \mathrm{deg}$ were tested. The range of reduced frequencies tested for the plunging cases was the same as the range for the plunging cases.

For the simulations, wing discretizations of 15 spanwise by 5 chordwise rings and 30 spanwise by 10 chordwise rings were evaluated. In Figs. 9a-9c the lift data are presented in terms of the magnitude of complex lift, which is equivalent to $L /(2 q b)$. The data are plotted as a function of $k$. Figures $9 \mathrm{a}$ and $9 \mathrm{~b}$ show that an increase in the number of rings used from 75 to 300 affords only slight improvement in the accuracy of the results.

Halfman $^{38}$ also provides data on the phase shift between wing position and maximum lift. Figures $10 \mathrm{a}-10 \mathrm{c}$ show that the phase results from the code are in good agreement with both theory and experimental data.

The time-dependent moments for these cases were also calculated and are compared to the experimental data. To determine these values, each case was run twice, using two different numbers of 


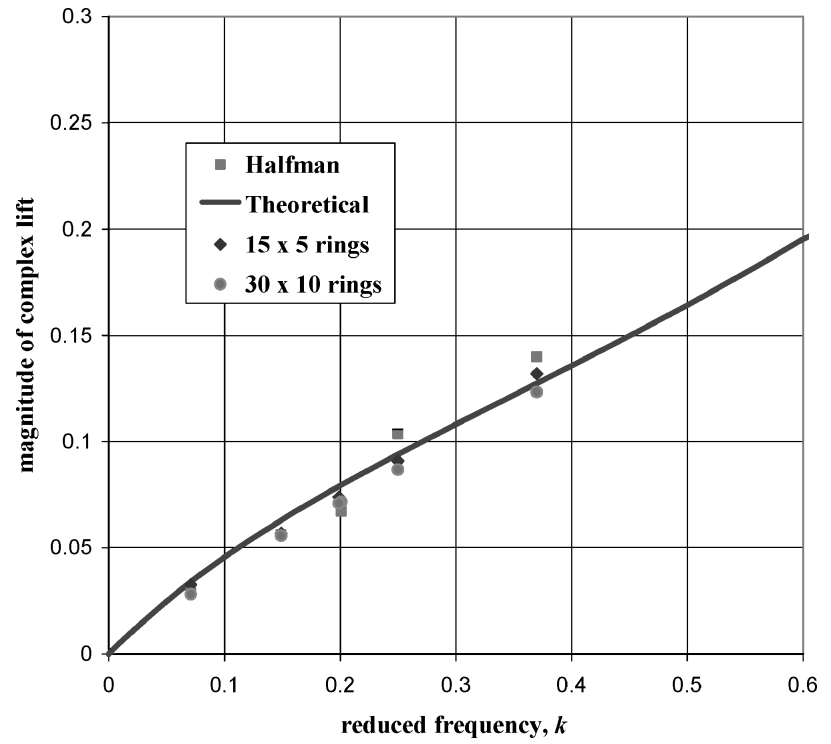

a)

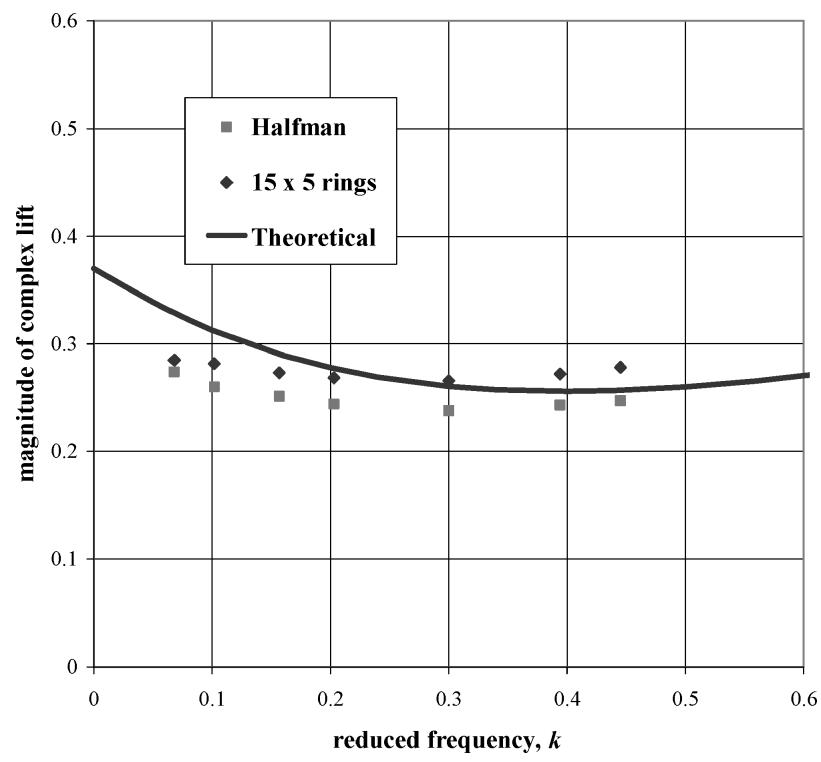

b)

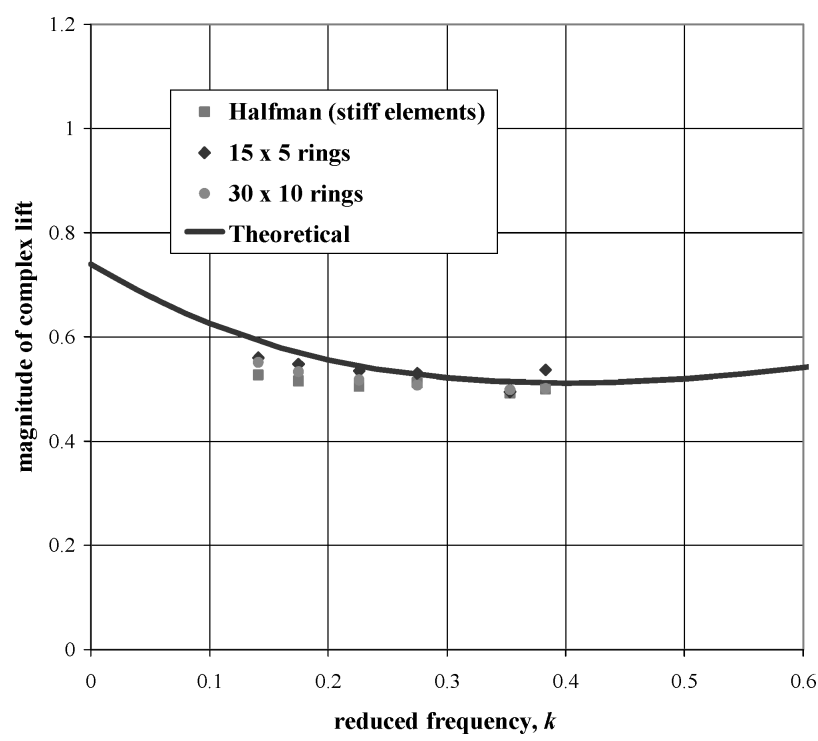

c)

Fig. 9 Experimental data compared to a) plunging case, $h_{0}=1$ in.; b) pitching case, $6.7 \mathrm{deg}$; and c) pitching case, $13.5 \mathrm{deg}$.

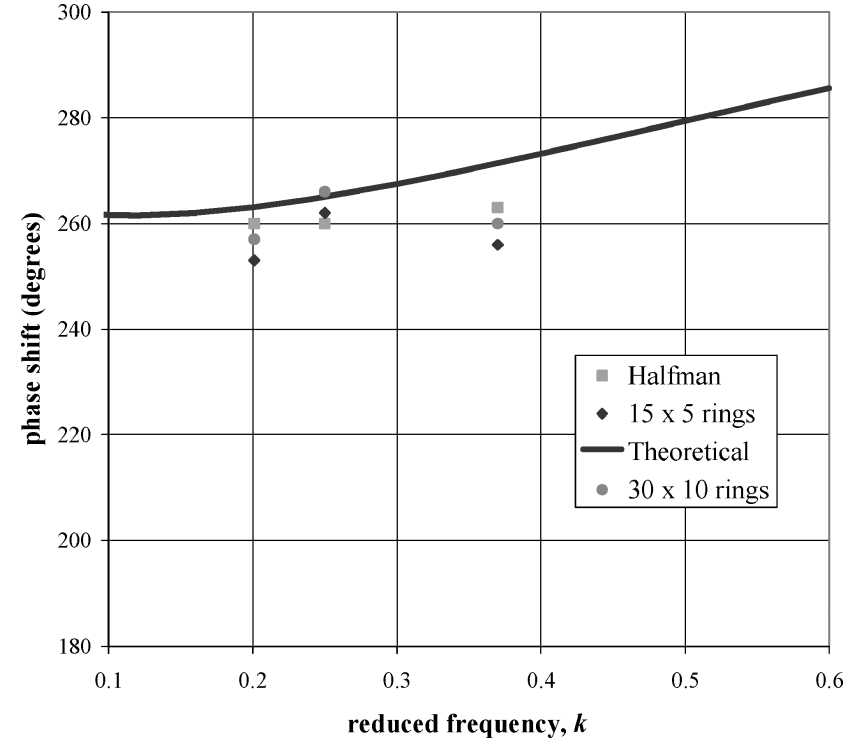

a)

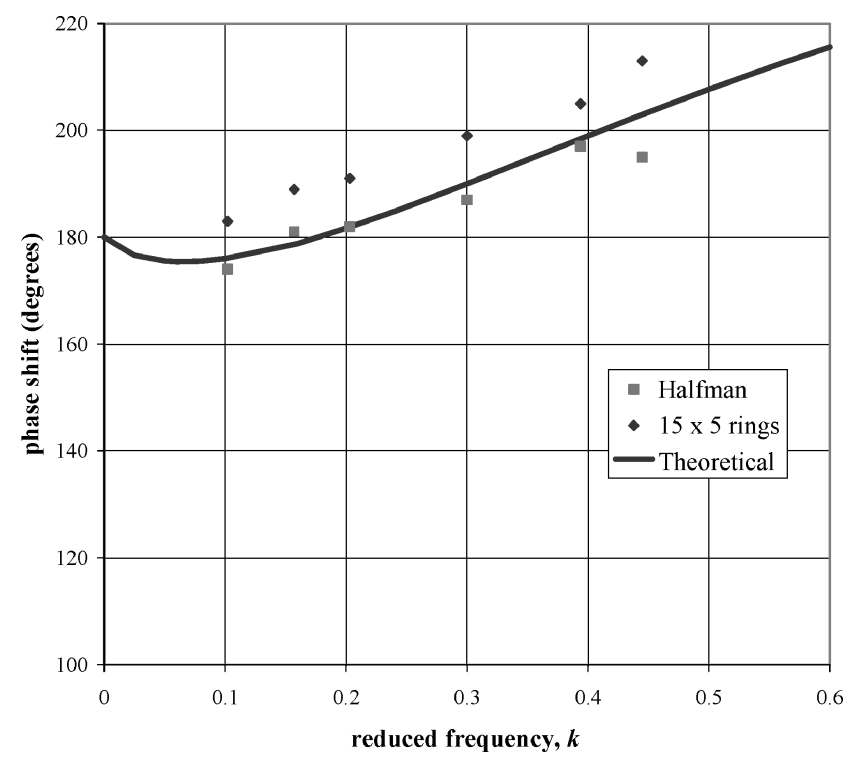

b)

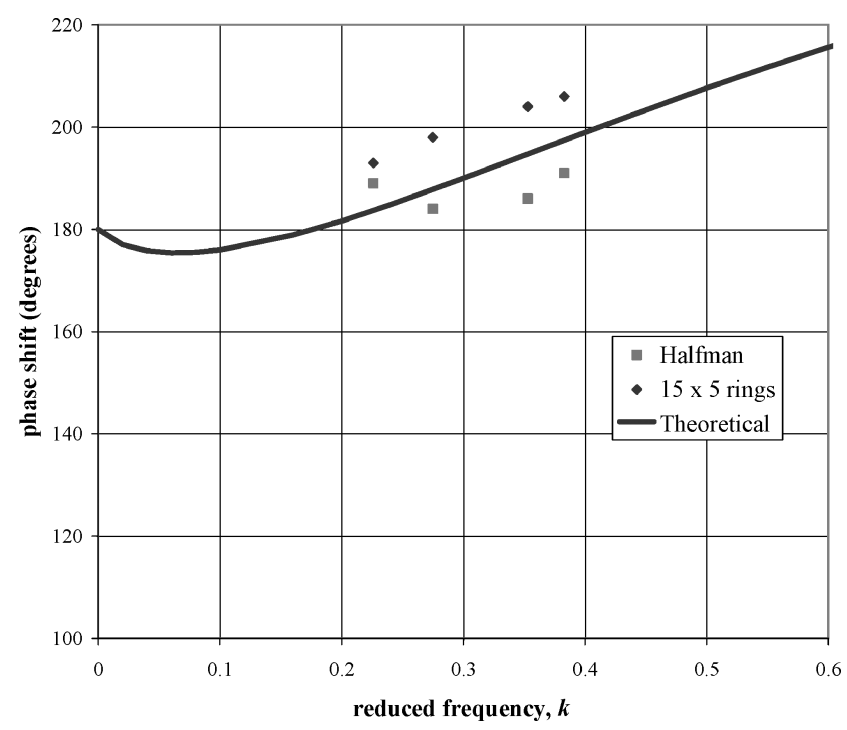

c)

Fig. 10 Phase shift for a) plunging case, $h_{0}=1$ in.; b) pitching case, $\alpha=6.74 \mathrm{deg}$; and c) pitching case, $\alpha=13.48 \mathrm{deg}$. 


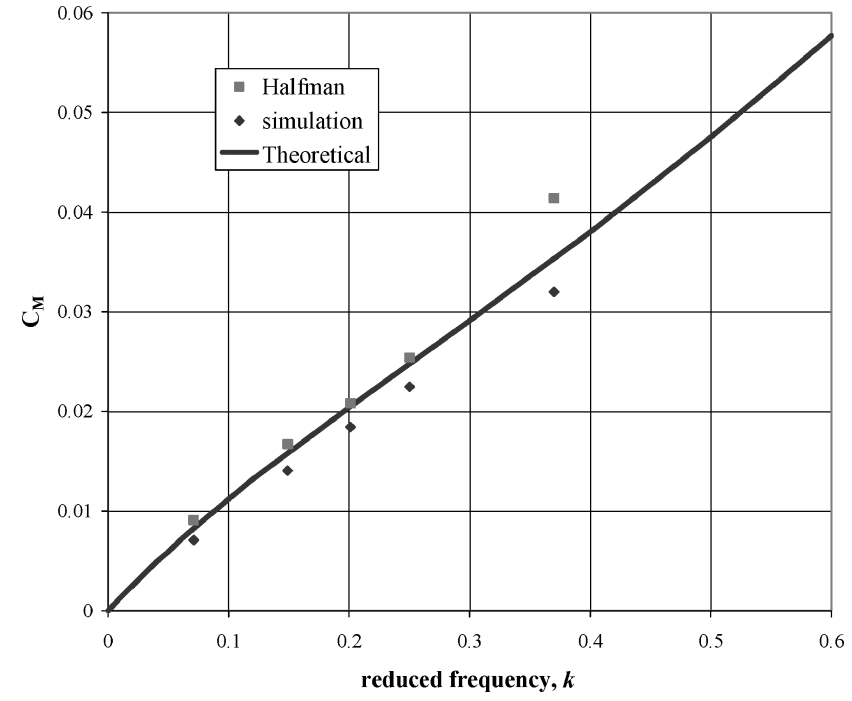

a)

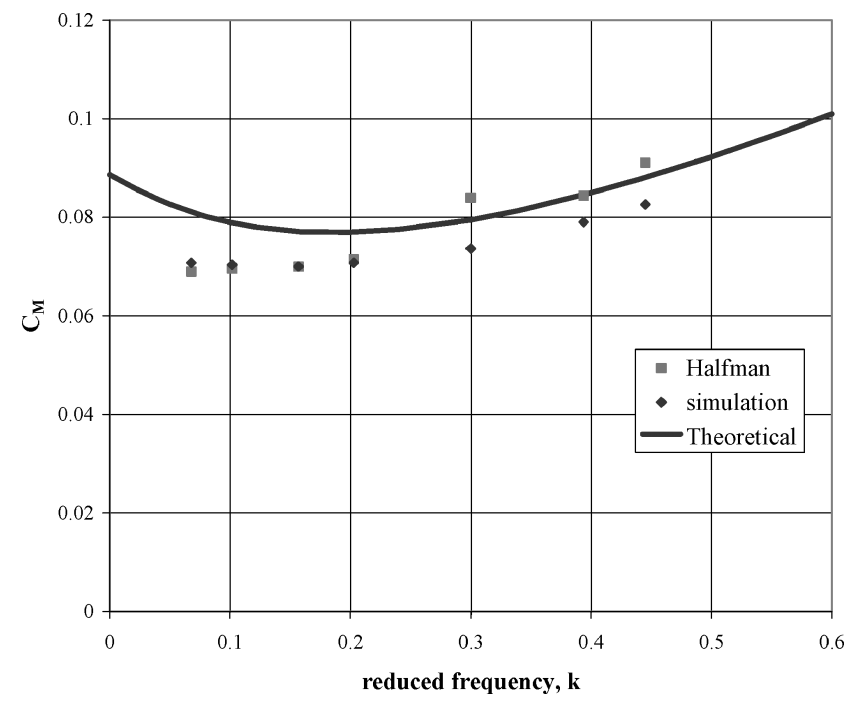

b)

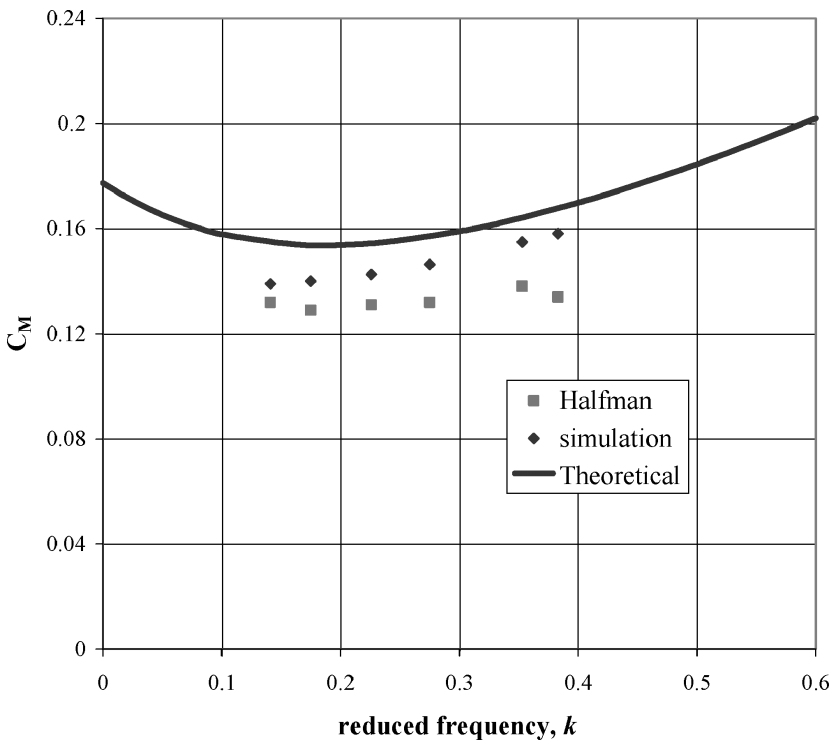

c)

Fig. 11 Moment coefficient for a) plunging case, $h_{0}=1$ in.; b) pitching case, $\alpha=6.74 \mathrm{deg}$; and c) pitching case, $\alpha=13.48 \mathrm{deg}$.

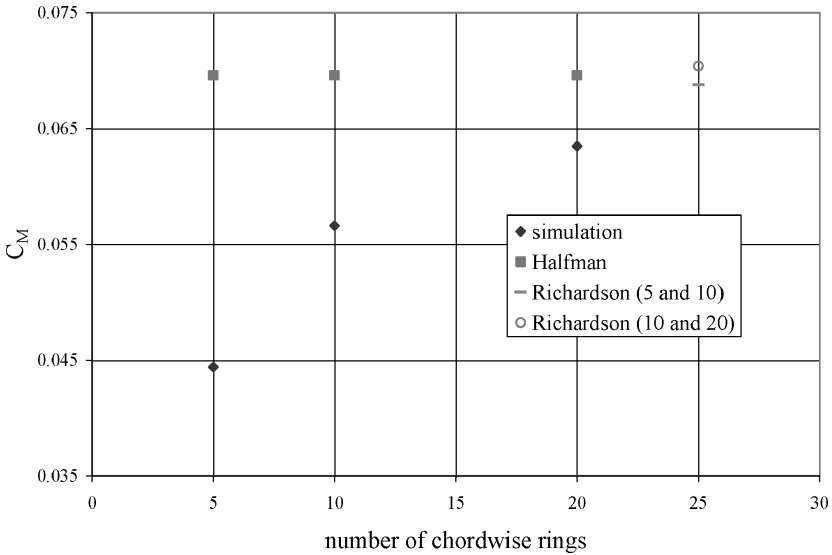

Fig. 12 Approximation of pitching moment for a pitching case where $k=0.102, \alpha_{0}=6.7 \mathrm{deg}$, using Richardson extrapolation.

wing-bound rings. The results were then combined using Richardson extrapolation (see Ref. 40) to increase accuracy. Figures $11 \mathrm{a}-11 \mathrm{c}$ show the results for pitching moment about the $37 \%$ chord location for the same cases shown in Figs. 9 and 10. The $C_{M}$ data are presented as a function of $k$. Again, these results are in good agreement with both experimental data and theoretical predictions.

Richardson extrapolation was applied to the simulation to make an accurate estimation of moment coefficient. The code was run twice for each of the simple harmonic oscillating cases, once using 5 chordwise wing-bound vortex rings and once using 10 rings. It was determined, after applying this technique with up to 20 chordwise rings, that an approximation based on a combination of 5- and 10ring runs was adequate, as shown by Fig. 12. (Note that magnitude of the error is exactly the same for both combinations shown.)

The theoretical values to which this work and Halfman's data ${ }^{38}$ are compared is based on the work of Theodorsen, ${ }^{39}$ a good summary of which can be found by Leishman. ${ }^{36}$ There are solutions for pure harmonic pitching and pure translation, and a combination of the two is simply a vector addition of them. ${ }^{38}$ The combined form of the two-dimensional lift equation can be written as

$$
\begin{aligned}
& L / 4 q b=-\pi\left[-k^{2} / 2+i k C(k)\right](h / b) \\
& -\pi\left\{\frac{1}{2}\left(i k+a k^{2}\right)+\left[1+i k\left(\frac{1}{2}-a\right)\right] C(k)\right\} \alpha
\end{aligned}
$$

where $C(k)$ is Theodorsen's function, which contains real and imaginary parts $F(k)$ and $G(k)$, respectively, $a$ gives the pitch axis location, $b$ is the semichord, and $\alpha$ and $h$ are the time-dependent angle of attack and plunge height, respectively. If the real part of lift is Re and the imaginary part is Im for a given case, then the expression for lift force is

$$
L / 4 q b=\sqrt{\operatorname{Re}^{2}+\operatorname{Im}^{2}} e^{i(\omega t+\phi)}
$$

where $\phi=\tan ^{-1}(\operatorname{Im} / \operatorname{Re})$.

The phase shift is, of course, given by $\phi$. The real and imaginary factors given by the theory for a two-dimensional wing can be found by Halfman. ${ }^{38}$ Similarly, the combined form of the two-dimensional moment equation is given by

$$
\begin{aligned}
& M / 4 q b^{2}=-\pi\left[\left(a k^{2} / 2\right)-\left(\frac{1}{2}+a\right) i k C(k)\right](h / b)-(\pi / 2) \\
& \quad \times\left\{\left[i k\left(\frac{1}{2}-a\right)-k^{2}\left(\frac{1}{8}+a^{2}\right)\right]\left(\frac{1}{2}+a\right)\left[1+i k\left(\frac{1}{2}-a\right)\right] C(k)\right\} a
\end{aligned}
$$

and expressed in terms of real and imaginary parts,

$$
M / 4 q b^{2}=\sqrt{\operatorname{Re}^{2}+\operatorname{Im}^{2}} e^{i(\omega t+\phi)}
$$

\section{Flapping}

After the investigation of harmonic pitching and plunging, the next step was to simulate flapping flight. Scientists interested in avian flight have proposed models for the primary aerodynamic quantities such as lift, thrust, and power for flapping flight, but 
Table 1 Comparison of steady lift coefficient between vortex lattice and Xfoil

\begin{tabular}{lccc}
\hline \hline Method/Airfoil & Conditions & $C_{l}{ }^{\mathrm{a}}$ & $C_{L}$ for finite span \\
\hline VLM & Inviscid & - & 0.660 \\
Xfoil (NACA 8306) & Inviscid & 0.891 & 0.686 \\
Xfoil (NACA 8306) & $R e=1 \times 10^{6}$ & 0.826 & 0.636 \\
Xfoil (NACA 8306) & $R e=5 \times 10^{5}$ & 0.813 & 0.626 \\
Xfoil (NACA 8318) & Inviscid & 0.987 & 0.760 \\
Xfoil (NACA 8318) & $R e=1 \times 10^{6}$ & 0.790 & 0.608 \\
Xfoil (NACA 8318) & $R e=5 \times 10^{5}$ & 0.752 & 0.579 \\
\hline \hline
\end{tabular}

${ }^{\mathrm{a}}$ From Xfoil. ${ }^{\mathrm{b}} A=8$.

few aerodynamicists have attempted to formulate rigorous computational simulation methods. Vest and Katz ${ }^{41,42}$ presented a model based on Ref. 29 that includes flapping and pitching motion of a bird's wings.

As a first step toward validating the present implementation, results are compared to Walker's theory. ${ }^{9}$ Though Walker's theory breaks down for higher-frequency flapping, it works well for low reduced frequencies. Osborne's theory ${ }^{13}$ (also see Ref. 4) introduces additional complexities that apply to hovering flight and insect flight. If an average position along the wing is assumed to be half the distance from the root to the tip, $l / 2$ (where $l$ is the distance from root to tip), and $\alpha=0$, Walker's equation for flapping lift coefficient can be expressed as

$$
C_{l}=\left[\sin \left(\beta_{0}\right) / \beta_{0}\right]\left(1+k_{W}^{2} / 8\right) C_{l, \text { steady }}
$$

where $\beta_{0}$ is the flap amplitude and $k_{W}=4 l \beta_{0} n / V_{\infty}$. Walker's theory assumes a constant flapping velocity rather than sinusoidal flapping. In the preceding equation, lift and drag are assumed to be the same during the upstroke and downstroke, and it is assumed that there is no spanwise twist in the wing. Walker's full theory can be applied to cases where there are both upstroke/downstroke variations and twist. The wing in the current model flaps sinusoidally so that $n$ (in the definition of $k_{W}$ ) is multiplied by $2 / \pi$ to obtain the average flapping frequency. Also, assuming an average position along the wing of $r /$ semispan $=\frac{1}{2}$ may be an oversimplification. Note that the flapping $C_{l}$ can be different from the steady $C_{l}$, even for very small $k_{W}$, when the flapping amplitude is large, due to the varying dihedral.

A highly cambered wing ( $8 \%$ camber with maximum camber at $30 \%$ chord, based on NACA 83XX airfoil) was modeled for different values of $k_{W}$ for comparison to Walker's theory. ${ }^{9}$ To find the steady lift coefficient, the same wing was first simulated with no flapping at zero angle of attack. This value was verified using the program $\mathrm{Xfoil},{ }^{43}$ to which a correction for finite aspect ratio was applied, as shown in Table 1. The viscous results and NACA 8318 results are included for completeness.

The equation used to correct for aspect ratio is

$$
C_{L_{\alpha}}=C_{l_{\alpha}}\{A /[A+2(A+4) /(A+2)]\}
$$

where $A$ is the aspect ratio. ${ }^{44}$ Note that for $A=8$, the finite span lift coefficient is equal to 0.77 times the two-dimensional lift coefficient. The VLM assumes an infinitely thin wing, whereas the Xfoil code can account for leading-edge suction, compressibility effects, viscous effects, and wing thickness. However, Xfoil can only be used for steady two-dimensional flows. The VLM result compares well with the corrected inviscid, $6 \%$ thick Xfoil result, as expected. The Xfoil results are used subsequently in Walker's theory.

Figure 13 shows that the code presented here agrees with Walker's theory where the theory is valid, that is, for small values of $k_{W}$. For values of $k_{W}$ higher than about 1 , the flapping frequency and amplitude are quite high (exceeding $20 \mathrm{~Hz}$ and $30 \mathrm{deg}$, respectively), and the forward velocity low. The influence of the vortex wake clearly has a big effect in such cases, as does the time variation of the velocity potential. Weis-Fogh and Jensen ${ }^{4}$ note that flapping will only contribute to lift if $k_{W}$ is greater than 0.66 , and the results of this code agree.

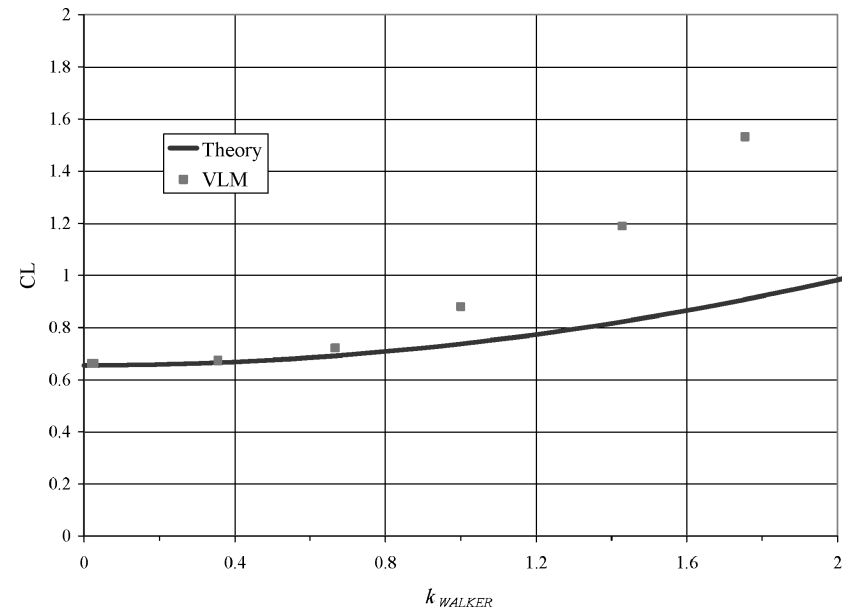

Fig. 13 Comparison of $C_{L}$ for different $k_{W}$ to Walker's theory; $\beta_{0}=15 \mathrm{deg}$.

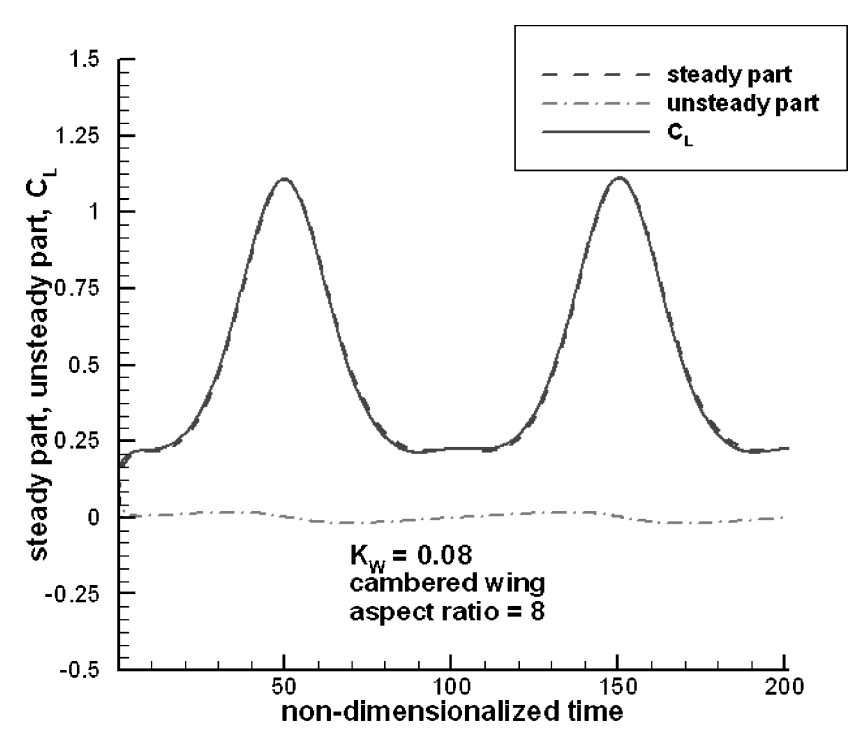

a)

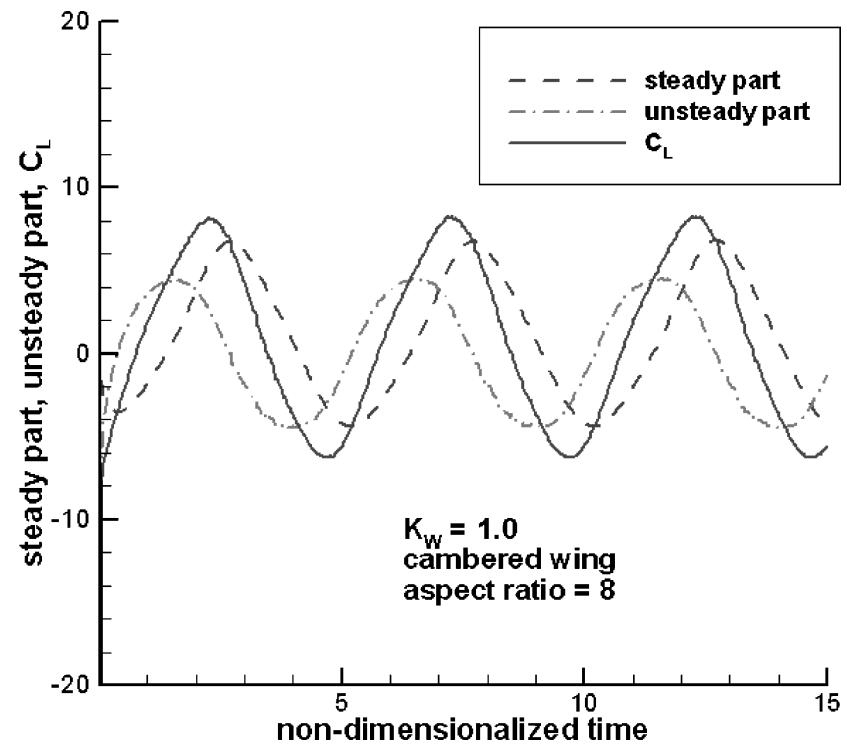

b)

Fig. 14 Contribution of steady and unsteady parts to total $C_{L}$ for two flapping cases: a) $k_{W}=0.08$ and b) $k_{W}=1.0$. 


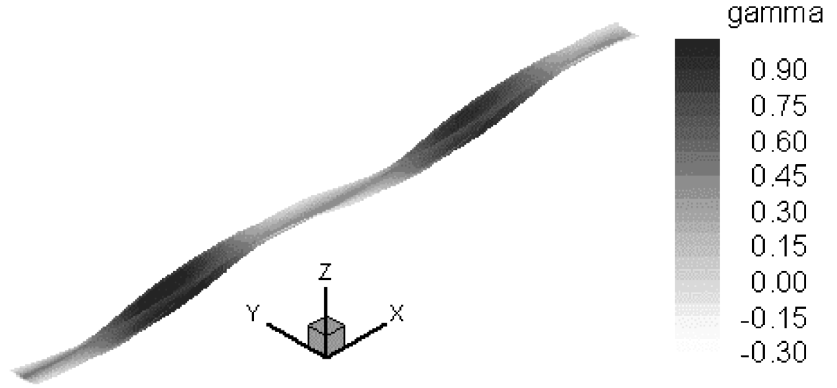

a)

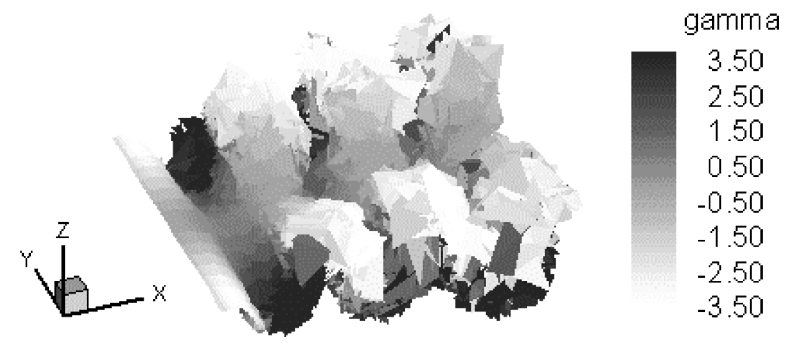

b)

Fig. 15 Wakes for flapping cases at two different flapping frequencies: a) $k_{W}=0.08$ and b) $k_{W}=1.0$

Figure 14 shows how the contribution of the unsteady part of $C_{L}$ [the time derivative of the velocity potential, the last term in Eq. (2)] to the total value increases with $k_{W}$ as predicted by the VLM code. For the case in Fig. 14a, $k_{W}$ is equal to 0.08 and the unsteady part of $C_{L}$ is approximately zero. For the case in Fig. $14 \mathrm{~b}, k_{W}$ is equal to 1.0 and the unsteady part of $C_{L}$ is contributing almost as much as the steady part. This result supports the idea that Walker's theory, ${ }^{9}$ which follows a quasi-steady approach, cannot be used to predict high-frequency flapping flight. These results also suggest that the high $C_{L}$ values calculated by some experimentalists may be correct. Figure 15 shows how the wakes for the two cases in Fig. 14 differ qualitatively. A comparison of the two shows how the $k_{W}=0.08$ case might be more representative of a bird in flapping flight with high forward speed, whereas the $k_{W}=1.0$ case represents something closer to hovering.

\section{Flapping and Twisting}

The next step in creating a more sophisticated model of flapping avian flight is to investigate the effect of flapping combined with dynamic twist (pitching). In this section, different kinematic parameters were varied to determine their effect on the lift and thrust of the wings. A survey of different flapping frequencies (given either by $\omega$ or $k$ ), pitch angles $\alpha$ (including twist), and flap angles $\beta_{0}$ was conducted for a cambered rectangular wing. For low-speed or high-frequency flapping flight, dynamic twist is essential to avoid conditions where massive flow separation might occur. In addition, the dynamic twisting must vary along the span. Consider the effective angle of attack of the flapping wing tip. The vector sum of freestream velocity and flapping velocity results in a relative angle of attack that can be very large. Thus, a geometric angle of attack that varies along the wing span is very important to maintaining lift and thrust in flight (and in maintaining attached flow). Dynamic twist makes sense intuitively and is of course employed by all kinds of flying creatures. ${ }^{19}$ While stressing the importance of three-dimensional vortex wake effects on flapping flight, the unsteady lifting line theory of Phlips et al. ${ }^{45}$ neglects twist in the wing. Vest and Katz ${ }^{41}$ investigate wing twist, but only for a very specific case using a pigeon wing model.

In this VLM, a linear dynamic twist is applied so that the geometric angle of attack $\alpha$ at any point along the wing is given by $\alpha=\alpha_{\text {base }}-\alpha_{0}(r / \mathrm{R}) \sin (\omega t)$, where $r / \mathrm{R}$ represents the fraction of wing span (root to tip) at a given spanwise location along the wing and $\alpha_{\text {base }}$ is some constant pitch angle applied to the entire wing.

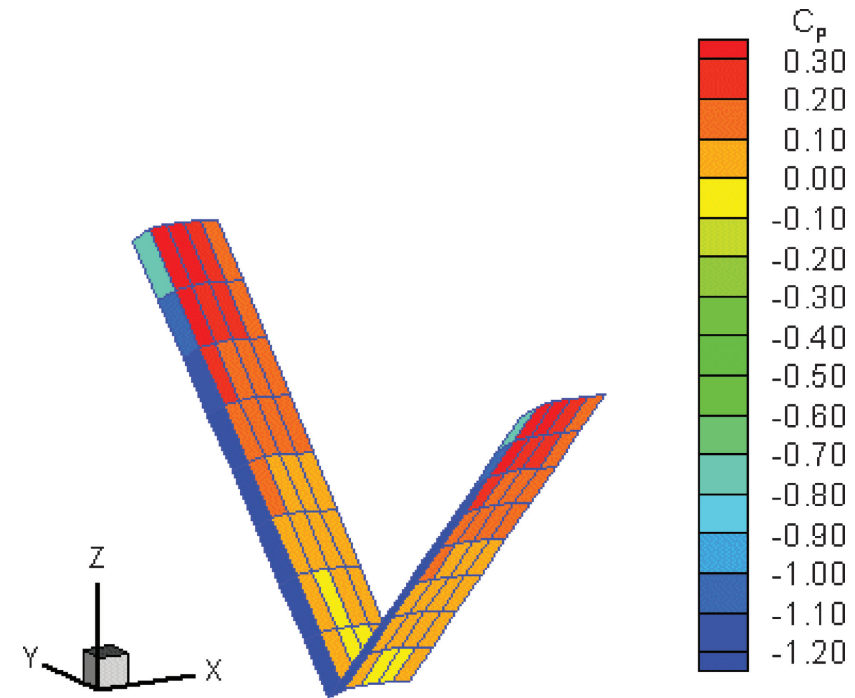

a)

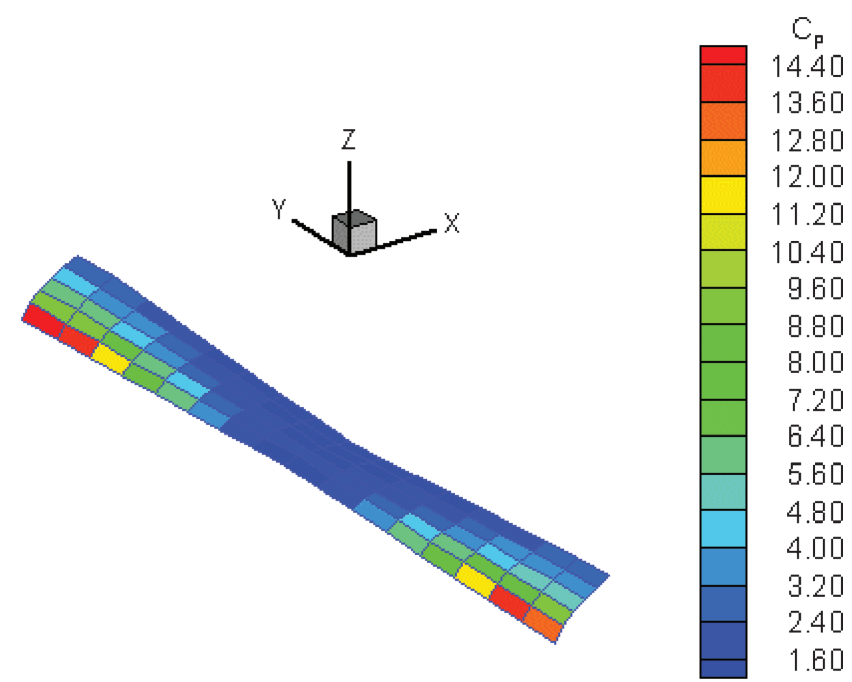

b)

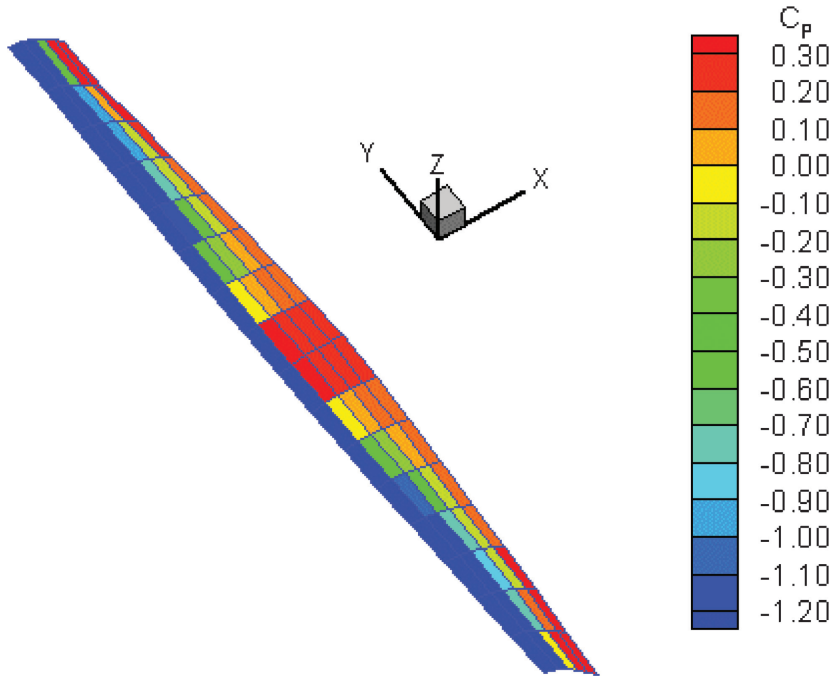

c)

Fig. 16 Snapshot of the dynamically twisting wing at various positions in the flap cycle; $\alpha_{0}=-41 \mathrm{deg}, \beta_{0}=45 \mathrm{deg}, \omega=55 \mathrm{rad} / \mathrm{s}$ (frequency $=8.8 \mathrm{~Hz}$ ), and $V_{\infty}=11 \mathrm{~m} / \mathrm{s}$ : a) tips-up (at the end of an upstroke), b) middownstroke, and c) midupstroke. 


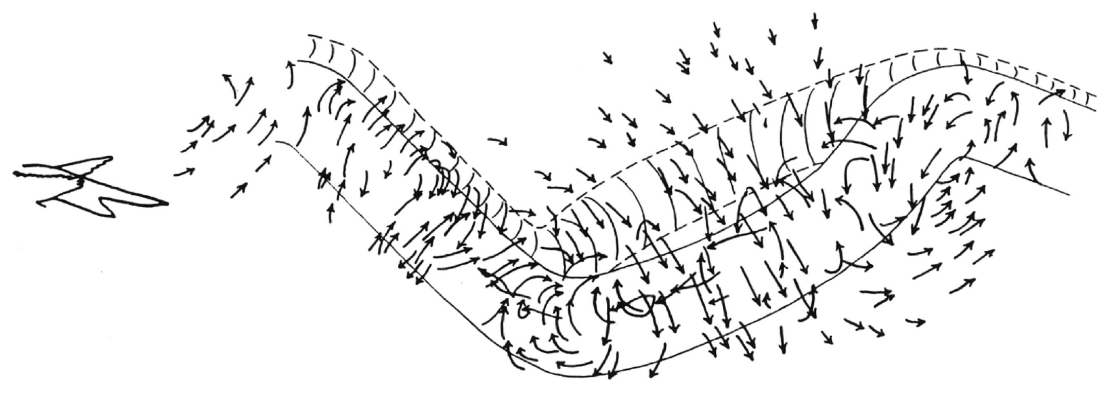

Fig. 17 Velocity vectors in the wake behind a Kestrel in flapping flight, $k=0.27$ (Ref. 23).
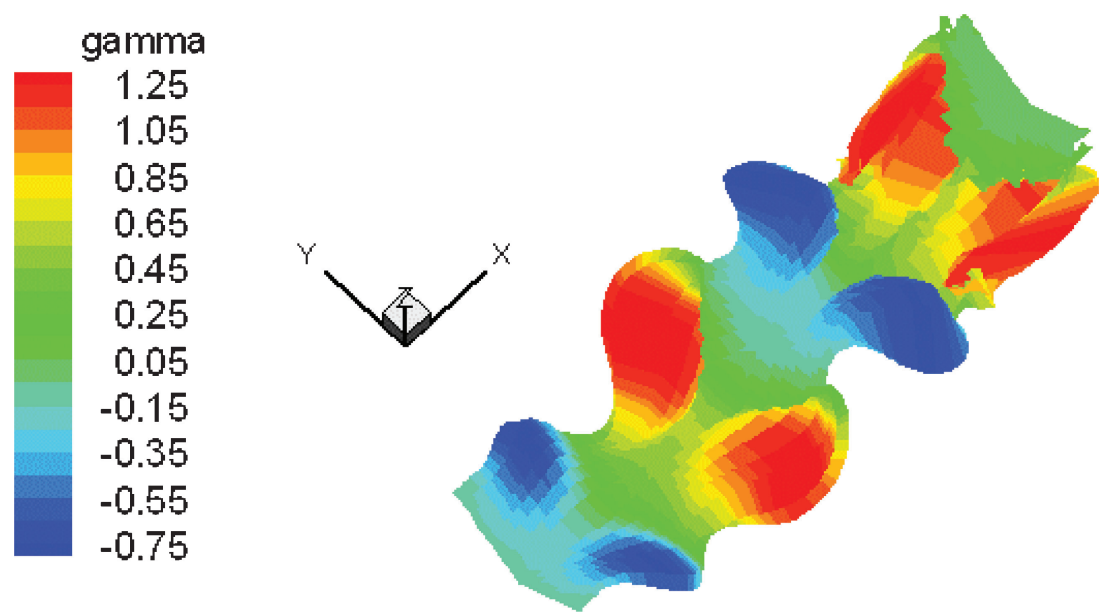

Fig. 18 Wake profile given by vortex lattice method, $k=0.19, \alpha_{0}=-14 \mathrm{deg}, \beta_{0}=45 \mathrm{deg}, \omega=55 \mathrm{rad} / \mathrm{s}(\mathrm{frequency}=8.8 \mathrm{~Hz})$, and $V_{\infty}=11 \mathrm{~m} / \mathrm{s}$.

The flap angle of the wing, $\beta$, oscillates at the same frequency as the pitch angle, where $\beta=\beta_{0} \cos (\omega t)$, although they are out of phase such that separation conditions are avoided. For the survey of kinematic flapping parameters, ranges of values for $\alpha_{0}, \beta_{0}$, and $\omega$ were varied one at a time, all other flight parameters being held constant, that is, wing geometry and freestream velocity.

Figure 16 shows the implementation of dynamic twist for this model. Figure 16a shows the position and pressure distribution over the wing at the wingtips-up position and Fig. 16b the wing at middownstroke. Figure 16c shows the wing midupstroke. Note how the orientation of each panel due to camber or twist combined with the sign of the pressure jump contribute to the sign and magnitude of the local lift and thrust.

The constant pitch angle $\alpha_{\text {base }}$ is applied to introduce asymmetry between the upstroke and the downstroke. Biologists ${ }^{19,27}$ have established that in the flapping cycle, particularly in low-speed (high reduced frequency) flight, thrust and lift are produced during the downstroke, whereas during the upstroke little useful force is generated. Birds may even pull their wingtips in or increase sweep angle, ${ }^{18}$ reducing span, drag, power expended, etc., during the upstroke. For a cambered airfoil such as the one investigated here, the zero-lift angle of attack is less than zero. To achieve an effective angle of attack near the zero-lift angle of attack on the upstroke and a higher effective angle of attack on the downstroke, a negative value for $\alpha_{\text {base }}$ was chosen.

The combined contributions of Rayner's ${ }^{12}$ highly sophisticated vortex model and the wake visualization experiments conducted by Spedding, along with the work of other biologists as discussed earlier (in "Biology-Based Work" section) give a fairly detailed picture of the wake of a bird in flapping flight. Figure 17 is from Spedding. ${ }^{23}$ Figure 17 is a result of his stereophotogrammetry work with a kestrel flying at a reduced frequency, $k=0.27$, and a medium speed, about $7 \mathrm{~m} / \mathrm{s}$. As a qualitative comparison, the wake found by the VLM for a case with a similar reduced frequency $(k=0.19)$ is shown in

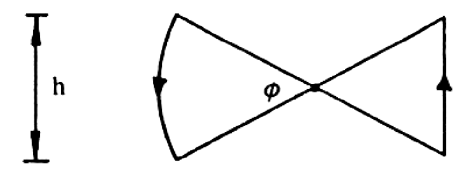

Head
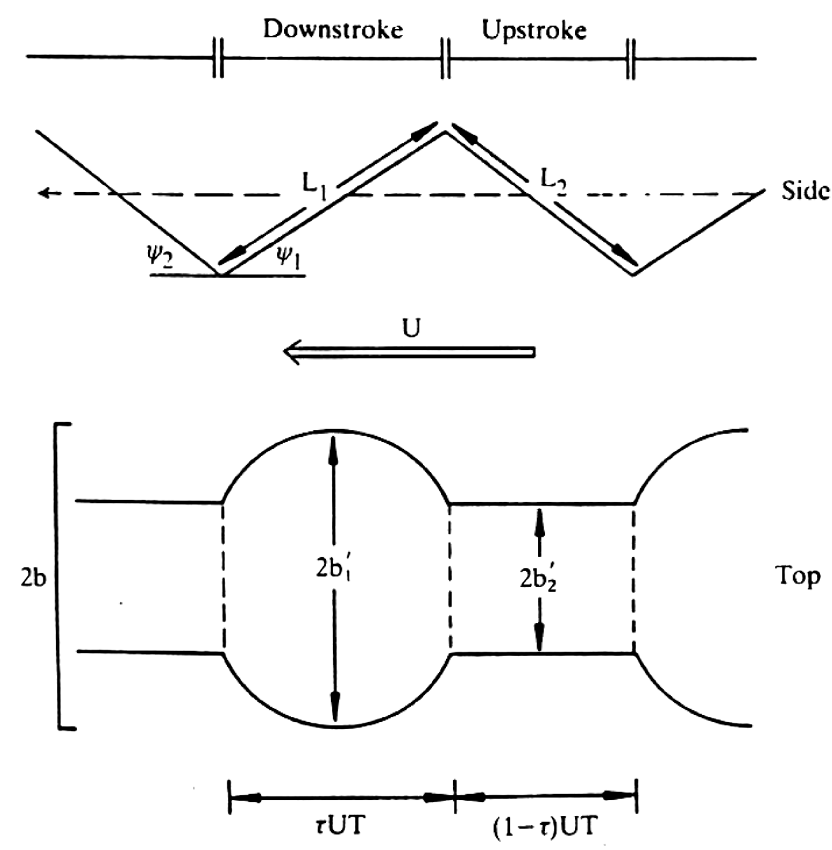

Fig. 19 Wake characteristics defined by Spedding (Ref. 23). 


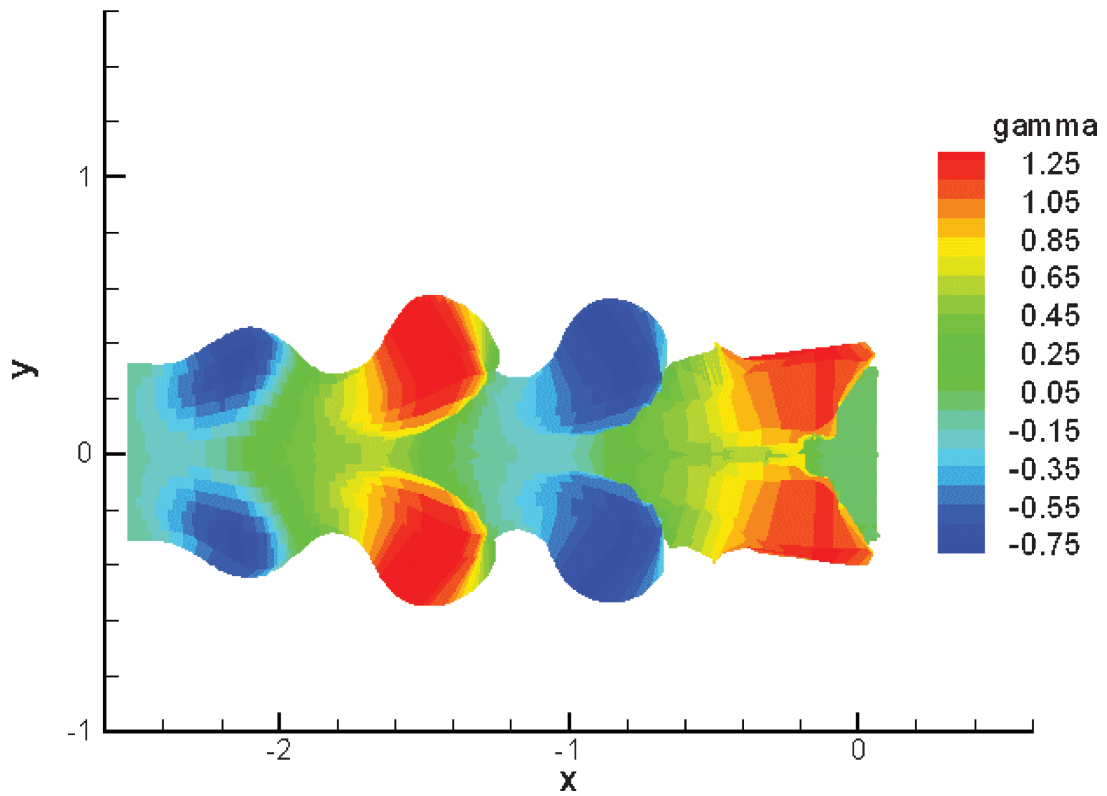

a)

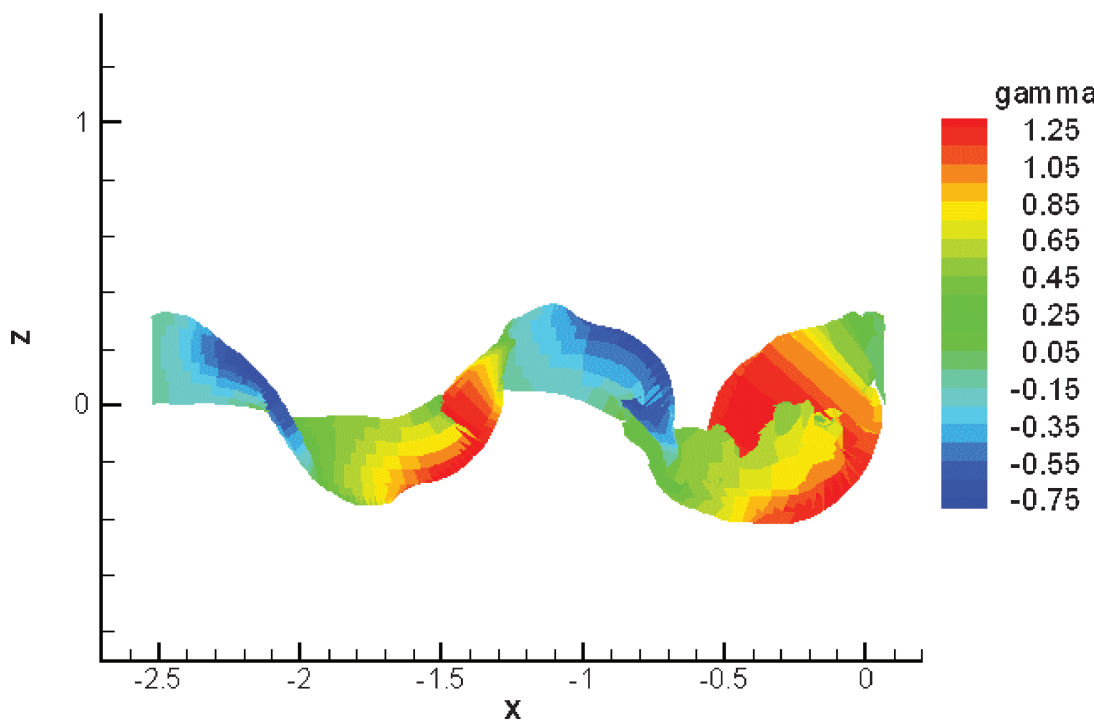

b)

Fig. 20 Wake shown in Fig. 18, $k=0.19$, units are meters: a) top and b) side.

Fig. 18. In Figs. 17 and 18, the bird or wing is traveling from right to left. Note that although the wing dimensions are similar, the details of the wing motions and shapes are different for the two cases. However, there is good qualitative agreement between the Fig. 17 from Spedding and the vortex lattice code. Figure 19, also from Spedding, ${ }^{23}$ shows how features of the wake can be characterized parametrically. Such quantities are easily calculated using results from the vortex lattice code, for example, as shown in Fig. 20. Again, Fig. 19 from Spedding shows good qualitative agreement with the computed wake.

Figure 21 shows the output for a typical flapping case with dynamic twist generated by the vortex lattice code over two flap cycles. For each case in the parametric survey, the wing was started from rest in the tips-up position so that each cycle began with a downstroke. Time-dependent $C_{L}, C_{M}$, and thrust data provide insight into the function and unsteady aerodynamics of each part of the flap cycle. For instance, Fig. 21 shows that the downstroke is where most of the thrust and lift are produced for this particular case, whereas the upstroke provides no positive lift or thrust. The downstroke occurs for a time range of $0-18.0$, whereas the first upstroke is for time between 18.0 and 36.3. Furthermore, the results show that the negative

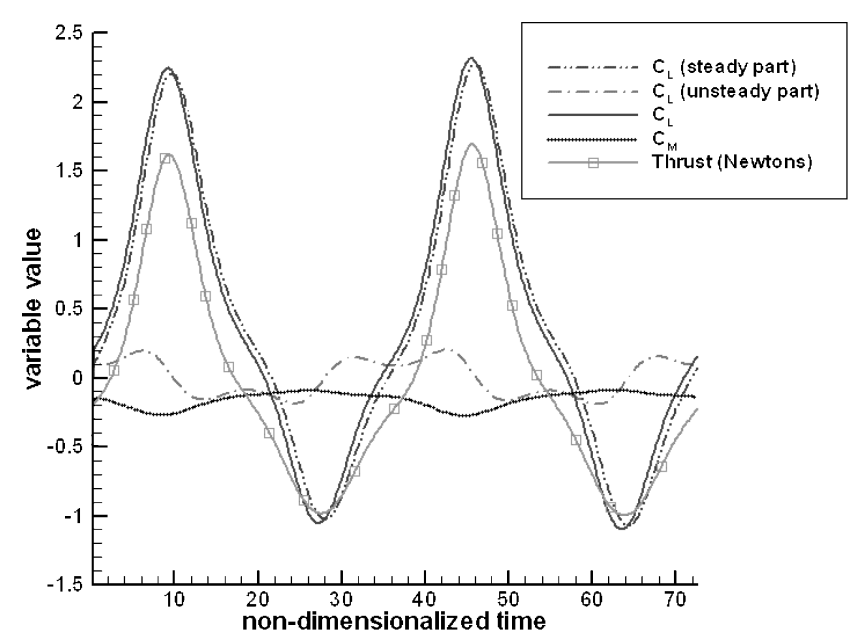

Fig. 21 Time-dependent lift, moment, and thrust data for $\alpha_{0}=5 \mathrm{deg}$, $\beta_{0}=45 \mathrm{deg}$, and $\omega=25 \mathrm{rad} / \mathrm{s}$. 
peaks in lift and thrust during the upstroke are smaller in magnitude than the positive downstroke peaks, suggesting a net positive thrust and lift over the complete cycle. It is expected that this phenomena would be even more pronounced were the wings to be swept back or pulled in during the upstroke as discussed earlier. Here the cambered airfoil and $\alpha_{\text {base }}$ are responsible for the asymmetry.

Table 2 gives the values of the parameters that were fixed for all cases evaluated. These values were chosen as representative of the case of a pigeon because the pigeon has been the subject of much

Table 2 Constant flight parameters

\begin{tabular}{lc}
\hline \hline Parameter & Value \\
\hline$V_{\infty}$ & $11.0 \mathrm{~m} / \mathrm{s}$ \\
$b$ (tip-to-tip) & $0.89 \mathrm{~m}$ \\
$c$ & $0.08 \mathrm{~m}$ \\
$\alpha_{\text {base }}$ & $3 \mathrm{deg}$ \\
Camber & NACA $83 X X$ \\
\hline \hline
\end{tabular}

study by Spedding, ${ }^{23}$ Rayner, ${ }^{27}$ Pennycuick, ${ }^{18}$ and others. The reader is cautioned, however, not to make comparisons between the results presented here to the actual lift and thrust of a pigeon because the geometry and kinematics of the simulated wing are not intended to duplicate exactly those of the real bird. Figures 22-24 show the trends in averaged quantities (thrust and lift) over one entire flap cycle, as well as over the up- and downstrokes alone. This information alone cannot fully represent the unsteady aerodynamics, which requires examination of the time-dependent force and moment profiles and the pressure distribution over the wing at any given moment in time (which are readily available as output from the code).

Notice that in Fig. 23a the average lift during the downstroke is less than the average lift on the upstroke and even falls below zero for low flap angles. In Fig. 23b, a negative thrust, that is, drag, is observed over most of the range of flap angles for both the upstroke and the downstroke. For a bird in flapping flight, especially at high frequencies, positive lift and thrust are expected and specifically during the downstroke. The negative values here are due to a poor

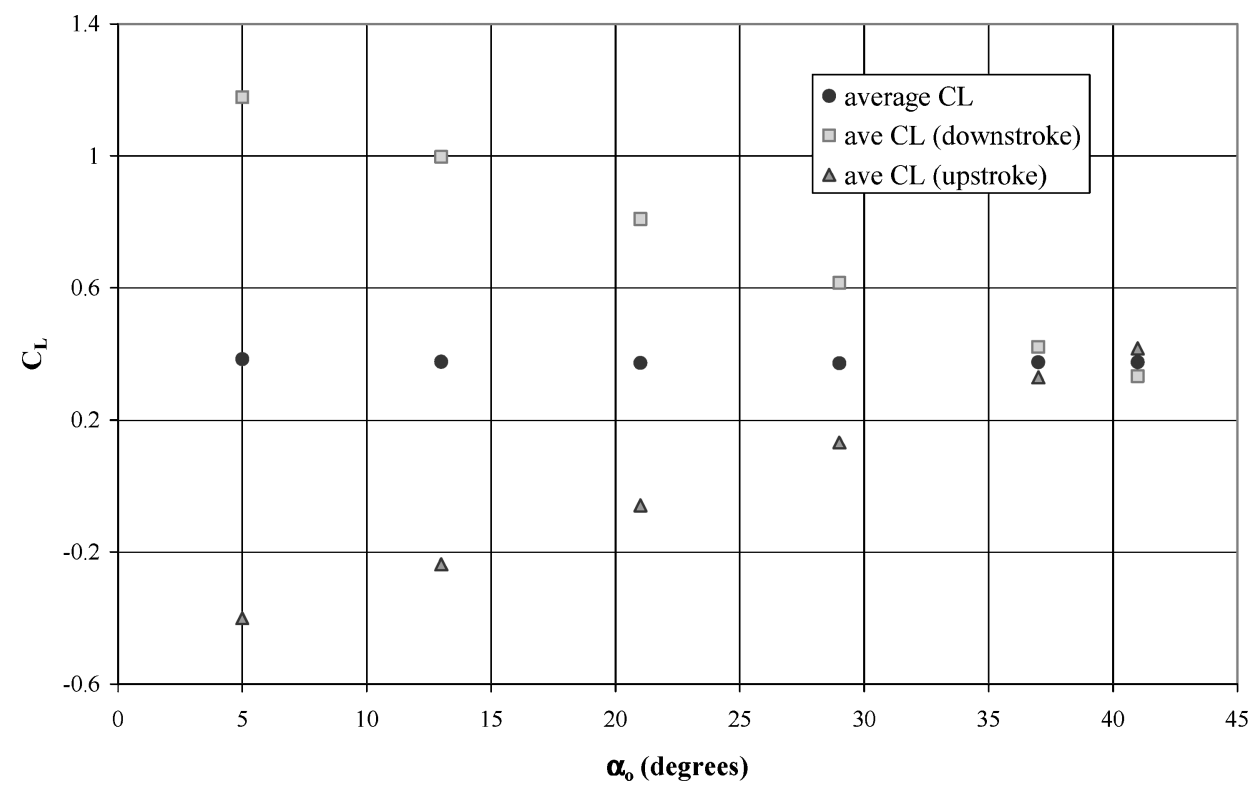

a)

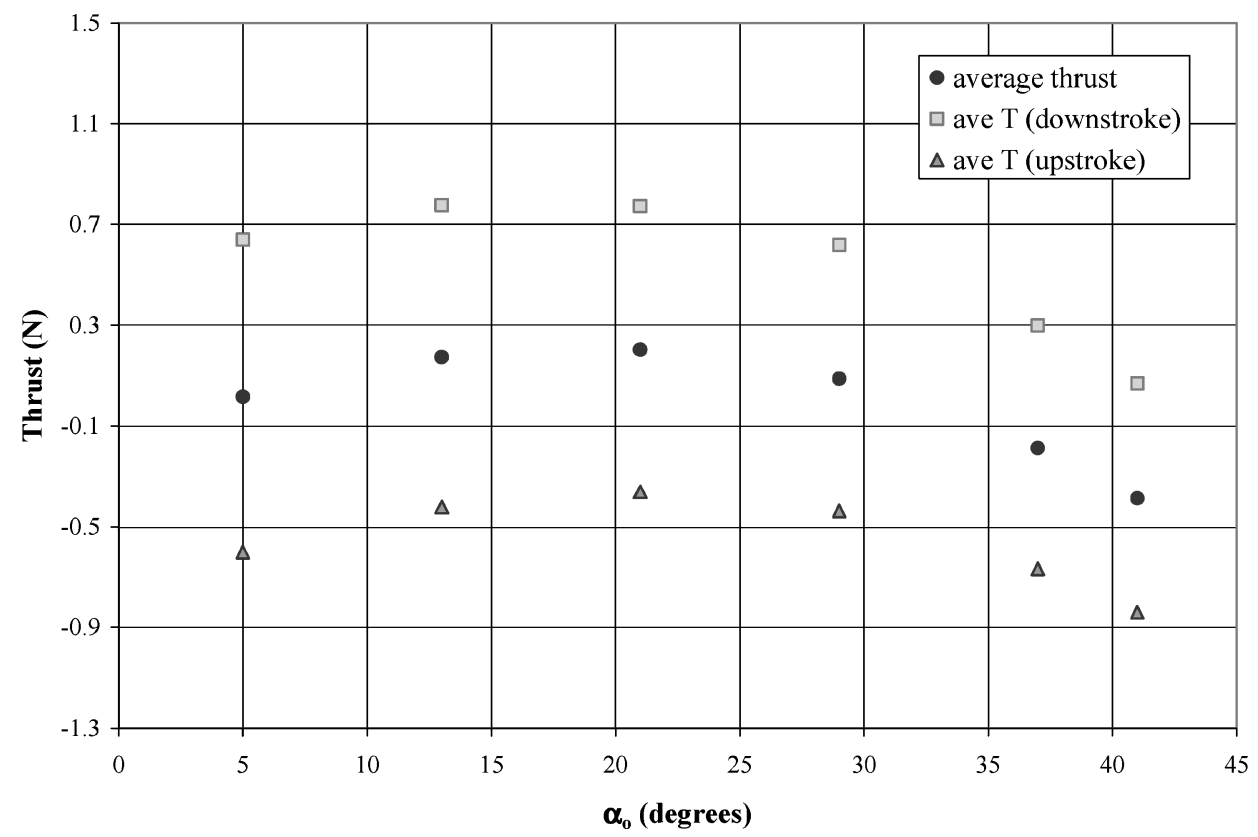

b)

Fig. 22 Various values of $\alpha_{0}, \omega=251 / \mathrm{s}$ and $\beta_{0}=45 \mathrm{deg}$ : a) average lift coefficient and b) average thrust. 


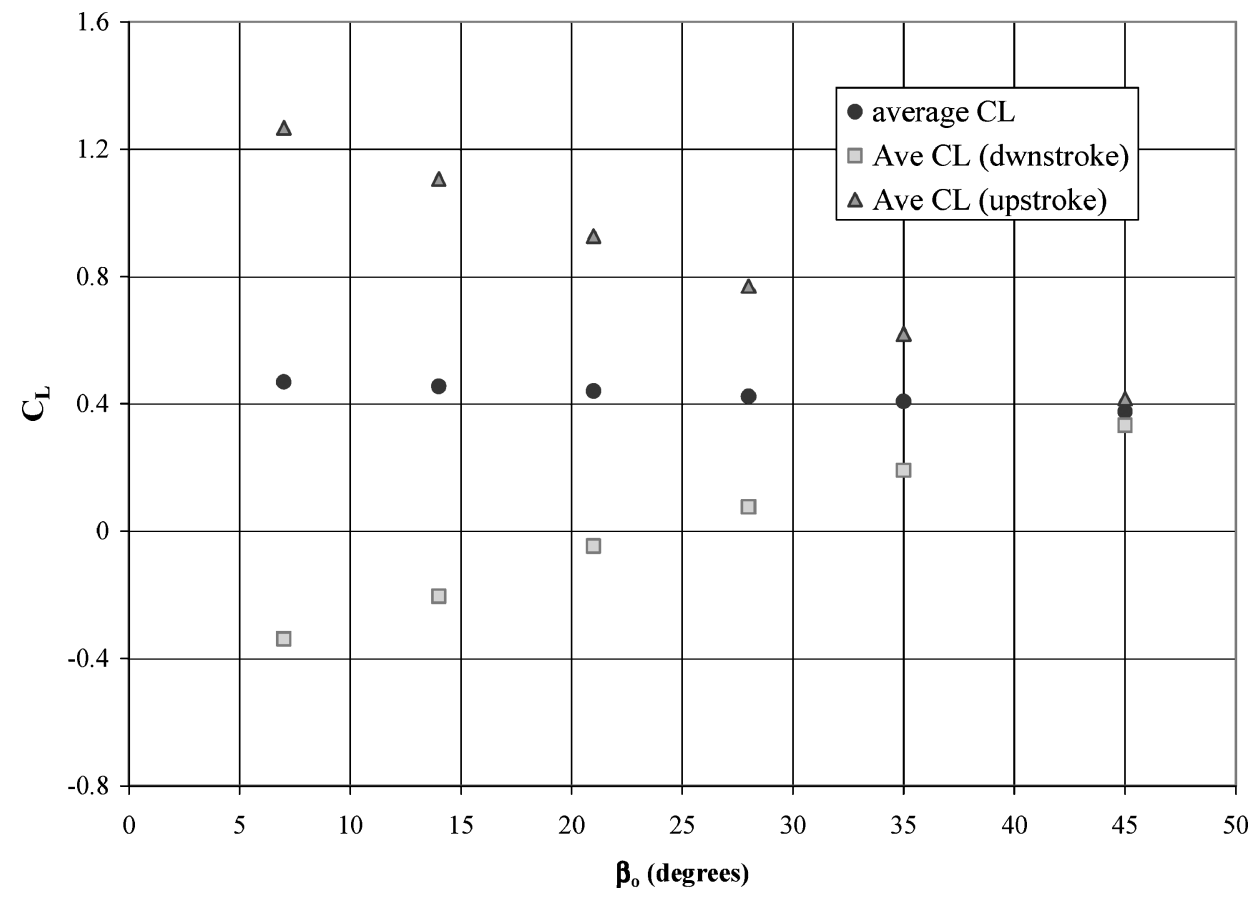

a)

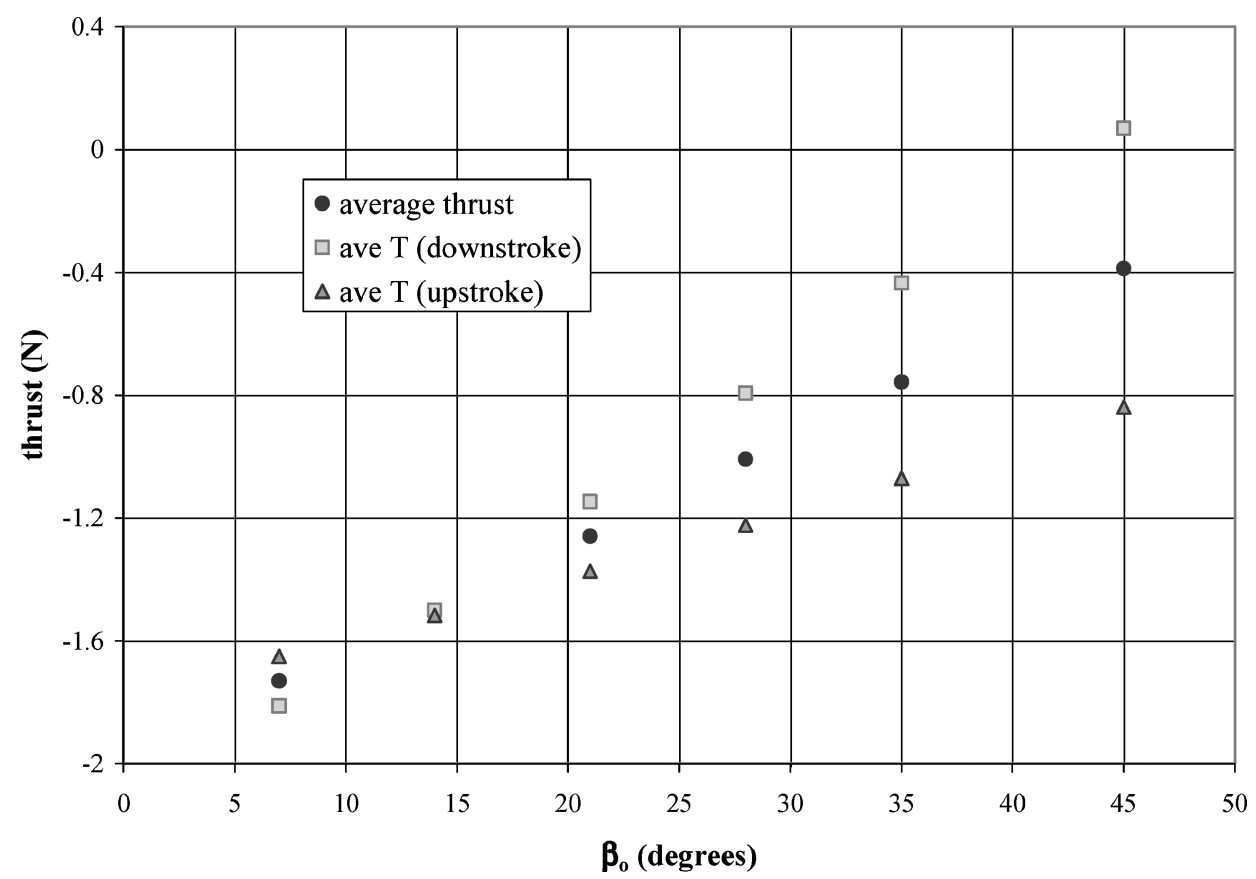

b)

Fig. 23 Various values of $\beta_{0}, \omega=251 / \mathrm{s}$ and $\alpha_{0}=41 \mathrm{deg}$ : a) average lift coefficient and b) average thrust.

choice of $\alpha_{0}$. The purpose of the wing twist, $\alpha_{0}$, is to counteract the detrimental effects of large effective angles of attack due to flap velocity. The flap velocity depends on $\beta_{0}$ if $\omega$ is fixed. Thus, for flap angles less than $45 \mathrm{deg}$ or so, such a large pitching angle will not produce a realistic lift or thrust profile, or more likely, massive separation will occur.

Studies of flapping avian flight at different speeds ${ }^{27}$ reveal that during high-speed, low-reduced frequency flight, lift may be produced during both the upstroke and downstroke of the flap cycle. Figure 24a supports this idea in general because lift produced during the downstroke increases as reduced frequency $(k \sim \omega)$ in- creases, whereas the relative importance of the downstroke compared to the upstroke is decreased for lower reduced frequencies. For the cases where $\omega$ is less than about $30 \mathrm{rad} / \mathrm{s}$, there is a positive lift for both the upstroke and the downstroke. For the lowest $\omega$ cases, unsteady effects are less important, and the quasisteady model would be a more appropriate analysis tool. Also as expected, the thrust produced during the downstroke in Fig. 24b increases steeply as $k$ increases, whereas the negative thrust (or positive drag) for the lower reduced frequencies are likely a reflection of unrealistic values of $\alpha_{0}$ and $\beta_{0}$ for such low reduced frequency conditions. 


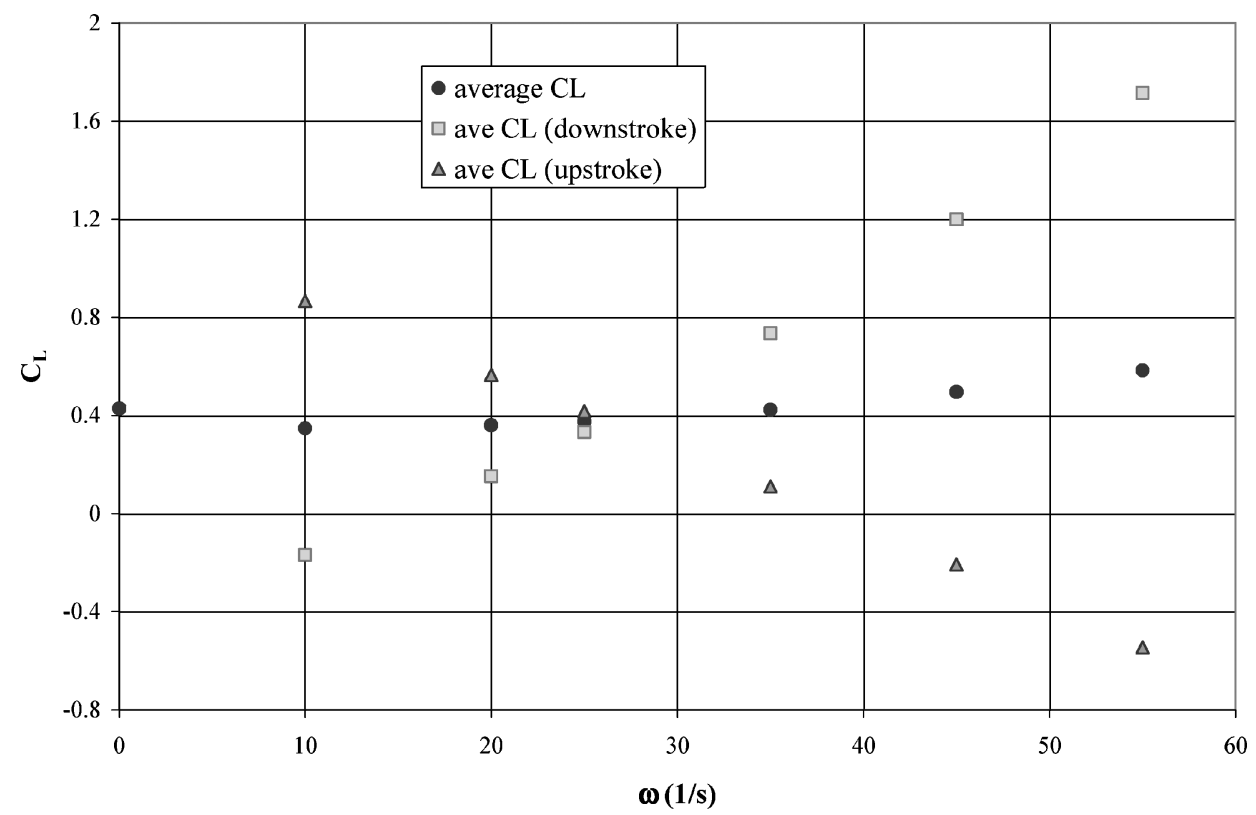

a)

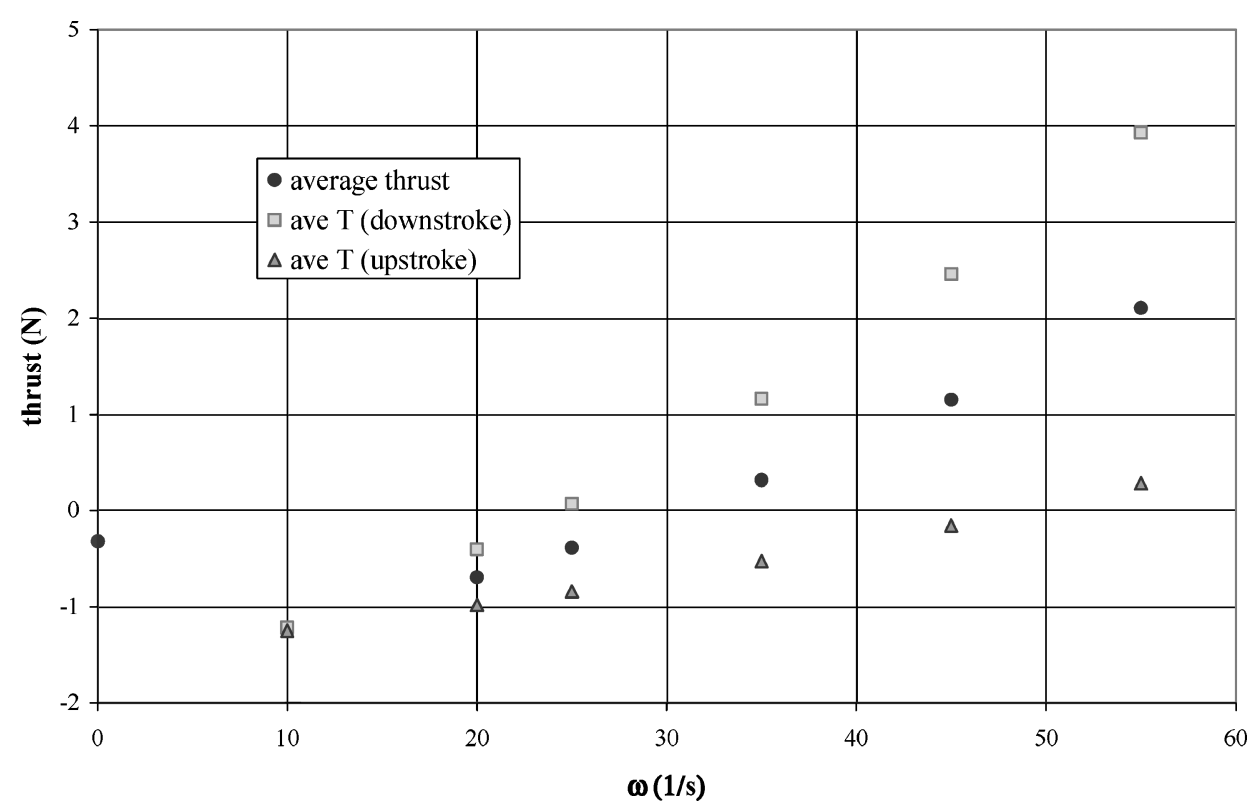

b)

Fig. 24 Various values of $\omega, \beta_{0}=45 \mathrm{deg}$ and $\alpha_{0}=41 \mathrm{deg}$ : a) average lift coefficient and b) average thrust.

\section{Conclusions}

The motivation for this study was to understand and predict the unsteady flapping flight of small birds and MAVs. A computational model for predicting the aerodynamics of high reduced frequency flapping flight has been developed. The foundation for the work has been laid by theory, experiments, and flow visualization performed by both biologists and aerodynamicists. Several analytical theories have been presented in the past with varying degrees of applicability to flapping flight aerodynamics.

For a wing at an angle of attack, impulsively started from rest, lift values including initial transient effects agree with results from Katz and Plotkin. ${ }^{29}$ Peak lift and phase shift values for oscillating plunging and pitching airfoils agree with the experimental data of Halfman ${ }^{38}$ and the theory of Theodorsen. ${ }^{39}$ The lift results for a flapping, cambered airfoil agree with Walker's theory ${ }^{9}$ within the range of reduced frequencies, where the theory is valid. This range is defined by the validity of the quasi-steady assumption and includes reduced frequencies less than about 1 . Adjustments for the camber and finite aspect ratio of the wing were made and were verified using the program Xfoil. When dynamic twist is added to the model for flapping cases, unsteady aerodynamic properties for conditions more closely representing the flight of actual birds can be calculated. A survey of the effect of three flapping parameters, flap angle, twist angle, and flapping frequency, revealed trends in lift and thrust averaged over the flapping cycle. Averages over the upstroke, downstroke, and overall cycle were considered. Insight into these trends can be gained by examining the pressure distribution over the wing at different points in the flapping cycle, as provided by the unsteady vortex lattice program. The model presented here has, thus, demonstrated its capability over a broad range of unsteady wing motions.

The current model uses an unsteady VLM that includes freewake relaxation with additional options for the purposes of accuracy (wake aging and stretching) and computational efficiency. In general, VLMs can achieve good accuracy using few rings; however, for complex surfaces and motions, more rings may be desirable. This model is implemented using object-oriented $\mathrm{C}++$, which makes the 
entire code much easier to read, maintain, and extend. Parallelization will further improve the code; an efficient parallel linear algebra solver, based on the CG method, was also developed. Parallelizing other parts of the code will greatly increase its capability and allow the simulation of hovering flight over many wingbeat cycles, which is the ultimate goal of the project. Results are very encouraging.

\section{Acknowledgments}

We would like to thank the National Science Foundation for funding the Consortium for Education in Many-Body Applications (http://www.cemba.psu.edu), Grant NSF-DGE-9987589.

\section{References}

${ }^{1}$ Jones, K. D., and Platzer, M. F., "Experimental Investigation of the Aerodynamic Characteristics of Flapping-Wing Micro Air Vehicles," Proceedings of the 41st AIAA Aerospace Sciences Meeting and Exhibit, Jan. 2003; also AIAA Paper 2003-0418, Jan. 2003.

${ }^{2}$ Weis-Fogh, T., "Energetics of Hovering Flight in Hummingbirds and in Drosophila," Journal of Experimental Biology, Vol. 56, No. 1, 1972, pp. 79-104.

${ }^{3}$ Weis-Fogh, T., "Unusual Mechanisms for the Generation of Lift in Flying Animals," Scientific American, Vol. 233, No. 5, 1975, pp. 81-87.

${ }^{4}$ Weis-Fogh, T., and Jensen, M., "Biology and Physics of Locust Flight. Basic Principles in Insect Flight, A Critical Review," Philosophical Transactions of the Royal Society of London, Series B, Vol. 239, No. 667, 1956, pp. 415-458.

${ }^{5}$ Wang, Z. J., "Vortex Shedding and Frequency Selection in Flapping Flight," Journal of Fluid Mechanics, Vol. 410, 2000, pp. 323-341.

${ }^{6}$ Ellington, C. P., "The Aerodynamics of Hovering Insect Flight, IV," Philosophical Transactions of the Royal Society of London, Series B, Vol. 305, Feb. 1984, pp. 1-144.

${ }^{7}$ Hall, K. C., and Hall, S. R., "Minimum Induced Power Requirements for Flapping Flight," Journal of Fluid Mechanics, Vol. 323, 1996, pp. 285-315.

${ }^{8}$ von Holst, E., and Küchemann, D., "Biological and Aerodynamical Problems of Animal Flight," Journal of the Royal Aeronautical Society, Vol. 46, 1942, pp. 39-56.

${ }^{9}$ Walker, G. T., "The Flapping Flight of Birds," Journal of the Royal Aeronautical Society, Vol. 29, 1925, pp. 590-594.

${ }^{10}$ Betteridge, D. S., and Archer, R. D., "A Study of the Mechanics of Flapping Wings," Aeronautical Quarterly, Vol. 25, No. 2, 1974, pp. 129-142.

${ }^{11}$ Rayner, J. M. V., "A New Approach to Animal Flight Mechanics," Journal of Experimental Biology, Vol. 80, No. 1, 1979, pp. 17-54.

${ }^{12}$ Rayner, J. M. V., "A Vortex Theory of Animal Flight," Journal of Fluid Mechanics, Vol. 91, No. 4, 1979, pp. 697-763.

${ }^{13}$ Osborne, M. F. M., "Aerodynamics of Flapping Flight with Application to Insects," Journal of Experimental Biology, Vol. 28, 1951, pp. 221-245.

${ }^{14}$ Mueller, T. J. (ed.), Fixed and Flapping Wing Aerodynamics for Micro Air Vehicle Applications, Progress in Astronautics and Aeronautics, edited by P. Zarchan, Vol. 195, AIAA, Reston, VA, 2001, p. 586.

${ }^{15}$ DeLaurier, J. D., and Harris, J. M., "Experimental Study of OscillatingWing Propulsion," Journal of Aircraft, Vol. 19, No. 5, 1982, pp. 368-377.

${ }^{16}$ Greenewalt, C. H., Hummingbirds, Dover, New York, 1990, p. 162.

${ }^{17}$ Leishman, J. G., "Challenges in Modeling the Unsteady Aerodynamics of Wind Turbines," Proceedings of the 40th AIAA Aerospace Sciences Meeting and Exhibit, Jan. 2002; also AIAA Paper 2002-0037, Jan. 2002.

${ }^{18}$ Pennycuick, C. J., "Power Requirements for Horizontal Flight in the Pigeon Columba livia," Journal of Experimental Biology, Vol. 49, No. 3, 1968, pp. 527-555.

${ }^{19}$ Norberg, U. M., "Vertebrate Flight," Zoophysiology, edited by S. D. Bradshaw, Vol. 27, Springer-Verlag, Berlin, 1990, p. 291.

${ }^{20}$ Chai, P., Chen, J. S. C., and Dudley, R., "Transient Hovering Performance of Hummingbirds Under Conditions of Maximal Loading," Journal of Experimental Biology, Vol. 200, No. 5, 1997, pp. 921-929.

${ }^{21}$ Chai, P., and Dudley, R., "Maximum Flight Performance of Hum- mingbirds: Capacities, Constraints, and Trade-Offs," American Naturalist, Vol. 153, No. 4, 1999, pp. 398-411.

${ }^{22}$ Chai, P., Chang, A. C., and Dudley, R., "Flight Thermogenesis and Energy Conservation in Hovering Hummingbirds," Journal of Experimental Biology, Vol. 201, No. 7, 1998, pp. 963-968.

${ }^{23}$ Spedding, G. R., "The Wake of a Kestrel (Falco tinnunculus)," Journal of Experimental Biology, Vol. 127, No. 1, 1987, pp. 45-78.

${ }^{24}$ Spedding, G. R., "The Wake of a Jackdaw (Corvus monedula) in Slow Flight," Journal of Experimental Biology, Vol. 125, No. 1, 1986, pp. 287-307.

${ }^{25}$ Kokshaysky, N. V., "Tracing the Wake of a Flying Bird," Nature, Vol. 279, 1979, pp. 146-148.

${ }^{26}$ Brodsky, A. K., "Vortex Formation in the Tethered Flight of the Peacock Butterfly (Inachis io) L. (Lepidoptera, Nymphalidae) and Some Aspects of Insect Flight Evolution," Journal of Experimental Biology, Vol. 161, No. 1, 1991, pp. 77-95.

${ }^{27}$ Rayner, J. M. V., "Form and Function in Avian Flight," Current Ornithology, Vol. 5, 1988, pp. 1-66.

${ }^{28}$ Tannehill, J. C., Anderson, D. A., and Pletcher, R. H., Computational Fluid Mechanics and Heat Transfer, 2nd ed., edited by W. J. Minkowycz and E. M. Sparrow, Computational and Physical Processes in Mechanics and Thermal Sciences Series, Taylor and Francis, Philadelphia, 1997, p. 792.

${ }^{29}$ Katz, J., and Plotkin, A., Low-Speed Aerodynamics, 2nd ed., edited by M. J. Rycroft and W. Shyy, Cambridge Aerospace Series, Vol. 13, Cambridge Univ. Press, Cambridge, England, U.K., 2001, p. 613.

${ }^{30}$ van der Vorst, H. A., "Efficient and Reliable Iterative Methods for Linear Systems," Journal of Computational and Applied Mathematics, Vol. 149, No. 1, 2002, pp. 251-265.

${ }^{31}$ Karniadakis, G., and Kirby II, R. M., Parallel Scientific Computing in C++ and MPI: A Seamless Approach to Parallel Algorithms and their Implementation, Cambridge Univ. Press, Cambridge, England, U.K., 2003, p. 616.

${ }^{32}$ Preidikman, S., "Numerical Simulations of Interactions Among Aerodynamics, Structural Dynamics, and Control Systems," Ph.D. Dissertation, Dept. of Engineering Mechanics, Virginia Polytechnic Inst. and State Univ., Blacksburg, VA, 1998.

${ }^{33}$ Greengard, L., and Rokhlin, V., "A Fast Algorithm for Particle Simulations," Journal of Computational Physics, Vol. 73, No. 2, 1987, pp. 325-348.

${ }^{34}$ Cheng, H., Greengard, L., and Rokhlin, V., "A Fast Adaptive Multipole Algorithm in Three Dimensions," Journal of Computational Physics, Vol. 155, No. 2, 1999, pp. 468-498.

${ }^{35}$ Barnes, J., and Hut, P., "A Hierarchical $\mathrm{O}(\mathrm{N} \log \mathrm{N})$ Force-Calculation Algorithm," Nature, Vol. 324, 1986, pp. 446-449.

${ }^{36}$ Leishman, J. G., "Principles of Helicopter Aerodynamics," edited by M. J. Rycroft and R. F. Stengel, Cambridge Aerospace Series, Vol. 12. Cambridge Univ. Press, Cambridge, England, U.K., 2000, p. 496.

${ }^{37}$ Shtern, V., Core $C++$, Prentice-Hall PTR Core Series, Prentice-Hall, Upper Saddle River, NJ, 2001, p. 1237.

${ }^{38}$ Halfman, R. L., "Experimental Aerodynamic Derivatives of a Sinusoidally Oscillating Airfoil in Two-Dimensional Flow," NACA Rept. 1108, 1952.

${ }^{39}$ Theodorsen, T., "General Theory of Aerodynamic Instability and the Mechanism of Flutter," NACA Rept. 496, 1935.

${ }^{40}$ Gerald, C. F., and Wheatley, P. O., Applied Numerical Analysis, 7th ed., Pearson/Addison-Wesley, Boston, 2004, pp. 325-328.

${ }^{41}$ Vest, M. S., and Katz, J., "Unsteady Aerodynamic Model of Flapping Wings," AIAA Journal, Vol. 37, No. 7, 1996, pp. 1435-1440.

${ }^{42}$ Vest, M. S., and Katz, J., "Aerodynamic Study of a Flapping-Wing Micro-UAV," AIAA Paper 99-0994, Jan. 1999.

${ }^{43}$ Drela, M., "XFOIL: An Analysis and Design System for Low Reynolds Number Airfoils," Conf. on Low Reynolds Number Airfoil Aerodynamics, Univ. of Notre Dame Press, South Bend, IN, June 1989.

${ }^{44}$ McCormick, B. W., Aerodynamics Aeronautics and Flight Mechanics, 2nd ed. Wiley, New York, 1995, p. 652.

${ }^{45}$ Phlips, P. J., East, R. A., and Pratt, N. H., "An Unsteady Lifting Line Theory of Flapping Wings with Application to the Forward Flight of Birds," Journal of Fluid Mechanics, Vol. 112, 1981, pp. 97-125. 